Escuela Técnica Superior de Ingeniería de Sistemas Informáticos Doctorado en Ciencias y Tecnologías de la Computación para Smart Cities

Universidad Politécnica DE MAdrid
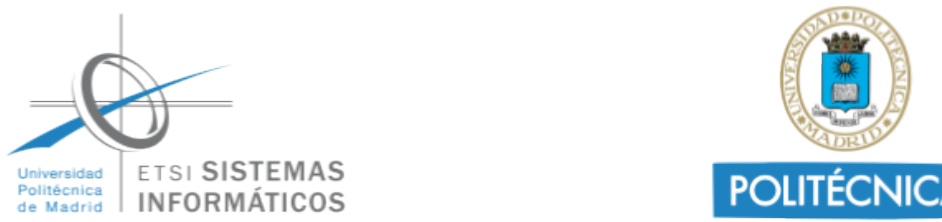

POLITÉCNICA

\title{
ESTRUCTURA DE CICLOS Y VÉRTICES en Digrafos Fuertemente Conexos Minimales
}

TESIS DOCTORAL

Autor: Miguel Arturo Arcos Argudo

Máster Universitario en Ciencias y TeCnologías de la

COMPUTACIÓN

DIRECTOR:

Jesús García López de Lacalle

Doctor en Matemáticas

Co-DiRector:

Luis Miguel Pozo Coronado

Doctor en Matemáticas

Madrid, 2020 


\section{Dedicatoria}

A mi esposa Kelly y a mi hijo David, no podría haber encontrado otra razón tan poderosa que me lleve a conseguir metas que pocos se atreven a buscar. La mitad de los méritos es suya. 


\section{Agradecimientos}

A Dios, a pesar de trabajar en el ámbito científico, soy de los que creen en su gracia, así que mi primer agradecimiento va para Él.

A mi esposa, por haber soportado los interminables días de ausencia, por las largas noches de trabajo, por haber tenido una paciencia enorme, y por la fuerza que cada día me transmite, sin importar la distancia.

A mi hijo, por aceptar nuestras despedidas, temporales pero largas, por haber cuidado de mamá cuando yo no estaba, por seguir siendo mi mejor amigo aunque se encuentre al otro lado del mundo, y por darme tan grandes lecciones, enseñándome, tal vez sin darse cuenta, que cualquier dificultad se puede superar.

A la Universidad Politécnica Salesiana

A Jesús, el Director de este trabajo, por guiarme, por escucharme, por haber creído en mi para elaborar esta tesis y, por supuesto, gracias por su amistad.

A Luis, el Co-director, desde hace mucho se convertió en lo más cercano a un familiar que pude haber tenido en Madrid, gracias por estar tan pendiente de mí, por tu esfuerzo entregado de manera tan generosa.

A mis padres, por haberme enseñado desde niño el valor de estudiar, por estar pendiente de mi familia cuando yo no estuve y por siempre esperar mi regreso.

Finalmente, a mis hermanas, mis cuñados, mi suegro, mis sobrinos, mis amigos y a mis compañeros de trabajo, porque cada uno de ustedes, en algún momento, me escucharon para compartir aunque sea una pequeña parte de toda esta larga aventura. 


\section{Índice general}

Dedicatoria

Agradecimientos

Índice general III

Índice de figuras $\quad \mathrm{V}$

Índice de tablas

Resumen 1

$\begin{array}{lr}\text { Abstract } & 2\end{array}$

1. Introducción 3

1.1. Objetivos ....................... 7

2. Introducción teórica $\quad 8$

2.1. Nociones básicas. Notación . . . . . . . . . . . . . . . . . . 8

2.2. Estado del arte . . . . . . . . . . . . . . . . . 20 20

3. Estructura de ciclos y vértices en los MSD 30

3.1. Estructura de ciclos . . . . . . . . . . . . . . . . . . . 30

3.1.1. Configuración de las SC ancladas . . . . . . . . . . . . 31

3.1.2. Consecuencia: Acotación del número de vértices lineales . . 33

3.2. Estructura de vértices . . . . . . . . . . . . . . . . . . . 43

3.2.1. Acotación del número de vértices lineales . . . . . . . . . . 44

3.2.2. Consecuencia en la longitud de los ciclos . . . . . . . . . . 48

3.3. Otros resultados . . . . . . . . . . . . . . . . . . . . . . . . . . . 49

3.3.1. Cadenas lineales externas . . . . . . . . . . . 50 
3.3.2. Coeficientes del polinomio característico . . . . . . . . 51

3.3.3. Algoritmos de búsqueda de ciclos de longitud máxima . . . 52

4. Conclusiones $\quad 54$

$\begin{array}{ll}\text { Bibliografía } & 56\end{array}$ 


\section{Índice de figuras}

2.1. Ejemplo de un grafo. . . . . . . . . . . . . . . 9

2.2. Ejemplo de un grafo conexo y ponderado. . . . . . . . . . . 10

2.3. Ejemplo de un digrafo conexo. . . . . . . . . . . . . . . . . 11

2.4. Ejemplo de un MSD de orden $n=18 \ldots \ldots \ldots \ldots \ldots$

2.5. Ilustración de dos MSD isomorfos. . . . . . . . . . . . . . . 13

2.6. Ejemplo de un grafo no dirigido de orden $n=7 \ldots \ldots \ldots$

2.7. Ejemplo de un MSD de orden $n=7 \ldots \ldots \ldots \ldots$

2.8. Ilustración de un digrafo conexo no fuertemente conexo $D_{1}$ y de un digrafo fuertemente conexo $D_{2} \ldots \ldots \ldots \ldots \ldots$

2.9. Ilustración de un árbol no dirigido $T$ y de un árbol dirigido doble $\overleftrightarrow{T}$ obtenido a partir de $T \ldots \ldots \ldots \ldots \ldots \ldots$

2.10. Construción de la primera oreja $\left(P_{0}\right)$ de un MSD. . . . . . 18

2.11. Construción de la oreja $P_{1} \ldots \ldots \ldots \ldots \ldots \ldots$

2.12. Construcción de un MSD mediante la adición de cada una de sus orejas. . . . . . . . . . . . . . . . . . . . . . 19

2.13. En el caso del MSD representado en (a) todos sus vértices pueden ser cubiertos mediante ciclos disjuntos. En el caso del MSD representado en (b) esto no es posible. . . . . . . . . . . . . 21

2.14. Ilustración de un ejemplo de árbol lineal $L_{4} \ldots \ldots$. . . . . . . 23

2.15. Ilustración de los tipos básicos de MSD lineales. . . . . . . . . . . 24

2.16. Ejemplo de MSD lineal formado por ciclos $C_{2} \ldots \ldots \ldots$. . . . 24

2.17. Ilustración de un ejemplo de MSD lineal. . . . . . . . . . . . . . 24

3.1. Ilustración del Lema 9. . . . . . . . . . . . . . . . . . . 32

3.2. Ilustración del Lema $10 \ldots$. . . . . . . . . . . . . . . . . . 33

3.3. Generación de una $\mathrm{SC}$ que contiene $p$ vértices de $C_{q} \ldots \ldots \ldots$

3.4. Ilustración del Lema 11. . . . . . . . . . . . . . . . . . . . . 38 
3.5. Ilustración del Teorema 1. . . . . . . . . . . . . . . . . . . . . . . 39

3.6. Ilustración del Lema 12. . . . . . . . . . . . . . . . . . . . . 40

3.7. Ilustración del Teorema 3. . . . . . . . . . . . . . . . . . . . . . 43

3.8. Ilustración de la Proposición 1. . . . . . . . . . . . . . . . . 46

3.9. Ilustración de la Proposición 2. Ejemplo de construcción de un MSD en el que existe un vértice contenido en todos los ciclos. . . 47

3.10. Ilustración de la ubicación de los vértices lineales en el MSD de la figura $3.9 . \ldots \ldots \ldots$. . . . . . . . . . . . . . . . . 48 


\section{Índice de tablas}

3.1. Número de configuraciones de $\mathrm{SC}$ del digrafo asociado a $\left(D, C_{q}\right) . \quad 36$ 


\section{Resumen}

Este trabajo muestra un estudio sobre la estructura de los ciclos contenidos en un Minimal Strong Digraph (MSD). La estructura de un ciclo dado está determinada por las componentes fuertemente conexas (strong components, o SC) que aparecen después de eliminar los arcos del ciclo. Mediante este proceso y mediante la contracción de todas las SC en un único vértice obtenemos un diagrama de Hasse que proviene del MSD. La estructura cíclica de esta familia de digrafos también puede estudiarse mediante el análisis de los vértices que tienen un alto grado de entrada o salida. Entre otras propiedades, demostramos que cualquier $\mathrm{SC}$ conformada por más de un vértice ( $\mathrm{SC}$ no trivial) tiene al menos un vértice lineal (un vértice con grado de entrada y grado de salida igual a 1) en el MSD; que en el diagrama de Hasse existe al menos un vértice lineal por cada maximal (minimal) no trivial (vértice con al menos un arco incidente); que si una SC contiene un número $\lambda$ de vértices del ciclo entonces contiene al menos $\lambda$ vértices lineales en el MSD; que dado un ciclo de longitud $q$ contenido en el MSD, el número $\lambda$ de vértices lineales contenidos en el MSD satisface $\lambda \geq\lfloor(q+1) / 2\rfloor$; pero también, hemos demostrado que $\lambda$ está acotado inferiormente por el grado máximo de entrada (o de salida) de cualquier vértice del MSD y que la máxima longitud de un ciclo contenido en el MSD es menor o igual a $2 n-m$, donde $n, m$ son el orden y el tamaño del MSD respectivamente; hemos encontrado una cota para los coeficientes del polinomio característico de un MSD, extendiendo el resultado presentado en [7]; y finalmente, hemos demostrado que el cálculo del ciclo con longitud máxima en un MSD es un problema NP-Duro. 


\section{Abstract}

This work shows a study about the structure of the vertices and cycles contained in a Minimal Strong Digraph (MSD). The structure of a given cycle is determined by the strongly connected components (SC, strong components) that appear after suppressing the arcs of the cycle. By this process and by the contraction of all SC in a unique vertex we obtain a Hasse diagram that comes from the MSD. The cyclic structure of this family of digraphs can also be studied by analyzing the vertices that have a high in- or outdegree. Among other properties, we show that any SC conformed by more than one vertex (non trivial SC) has at least one linear vertex (a vertex with indegree and outdegree equal to 1) in the MSD; that in the Hasse diagram exists at least one linear vertex for each non-trivial maximal or minimal (here, non-trivial means that the vertex has at least one incident arc); that if an SC contains a number $\lambda$ of vertices of the cycle then contains at least $\lambda$ linear vertices in the MSD; that given a cycle of length $q$ contained in the MSD, the number $\lambda$ of linear vertices contained in the MSD satisfies $\lambda \geq\lfloor(q+1) / 2\rfloor$; but also, we have proved that $\lambda$ is lower bounded by the maximal in- or outdegree of any vertex of the MSD and that the maximal length of a cycle contained in an MSD is lesser than or equal to $2 n-m$ where $n, m$ are the order and the size of the MSD respectively; we have found a bound for the coefficients of the characteristic polynomial of an MSD, extending the result in [7]; and finally, we have proved that computing the longest cycle contained in an MSD is a NP-Hard problem. 


\section{Capítulo 1}

\section{Introducción}

La teoría de grafos es un tema de interés para las Ciencias de la Computación. Su campo de aplicación incluye la modelización de estructuras de datos y el desarrollo algorítmico, lo cual ha permitido, por ejemplo, optimizar procesos y reducir costes en diferentes ámbitos.

Un grafo es una estructura discreta compuesta por vértices relacionados entre sí [30]. Su uso ha permitido modelar problemas en varios campos disciplinares, por ejemplo: relaciones entre personas en distintos contextos, colaboración entre investigadores, mapas de carreteras, sistemas ferroviarios, redes eléctricas, etc.

Algunos problemas algorítmicos clásicos se han modelizado mediante grafos, como el algoritmo de ordenación de complejidad óptima usando árboles binarios balanceados. Por otro lado, el ámbito de la Teoría de Grafos ha proporcionado muchos algoritmos interesantes, tales como el cálculo de circuitos eulerianos, cálculo de caminos hamiltonianos, la coloración de grafos, el problema del viajante, etc.

Todos los problemas mencionados existen en entornos reales y han podido ser modelados mediante el uso de grafos para su comprensión y posterior estudio. Es decir, existen varios problemas que inicialmente fueron planteados de una manera distinta, pero su comprensión y posterior resolución fue posible mediante su modelización con grafos. Por ejemplo, para el problema del ordenamiento ascendente de una lista de datos existen varias soluciones. Una de ellas consiste en colocar el primer dato de la lista en un nodo; el siguiente dato será colocado a la izquierda del primero, si es menor, o a su derecha, si es mayor. Para el resto de datos se realiza el mismo procedimiento de manera iterativa hasta encontrar su ubicación adecuada en la estructura de datos que se forma. Se obtiene como resultado final un grafo con forma de árbol, que puede ser recorrido mediante 
algoritmos sencillos de búsqueda en anchura y en profundidad. El ordenamiento y la búsqueda son problemas clásicos de algoritmia que se estudian en Ciencias de la Computación.

De manera similar al ejemplo anterior, existe una gran cantidad de casos en los que la teoría de grafos resulta ser altamente útil para la computación. A continuación se mencionan algunos:

- Los algoritmos que son implementados en un lenguaje de programación pueden ser representados mediante diagramas de flujo; éstos son un tipo de grafo que indica de manera visual la secuencia de acciones que deberá ejecutar el programa una vez que sea implementado.

- Una red de ordenadores puede tener distintas topologías, tales como: estrella, árbol, anillo, etc. y todas ellas, incluso Internet, pueden ser modelizadas mediante el uso de grafos; donde cada nodo respresenta un ordenador (o inclusive una subred) y cada arista la conexión entre dos nodos. Incluso es posible que la arista represente el sentido en el que fluye la información. En este contexto, cabe también mencionar la importancia de algoritmos útiles para las redes de ordenadores. Un ejemplo es el cálculo de la distancia entre dos nodos de la red, que se puede interpretar como el número de saltos que se deben dar para que un computador se comunique con otro. Otro ejemplo es la búsqueda de nodos de corte de la red, esto es, aquellos nodos que provocarían la desconexión de la red en el caso de que su funcionamiento se detuviese.

- Las bases de datos en las que empresas públicas y privadas guardan su información, organizan los datos en estructuras de árbol que soportan algoritmos eficientes de inserción, ordenamiento y búsqueda de datos.

- La programación distribuida consiste en un conjunto de ordenadores que ejecutan un mismo programa y cada uno de ellos contribuye con una parte del cálculo. Este paradigma se puede representar mediante grafos.

- Algunos famosos buscadores de internet se han servido de algoritmos inspirados en la teoría de grafos, como por ejemplo, el diseñado por Page et al. [40]. Con ello han logrado determinar el índice de importancia que tiene una página web y proporcionar mejores sugerencias a sus usuarios. 
- Los diagramas utilizados para el lenguaje de modelamiento unificado (UML, por sus siglas en inglés), tales como los diagramas entidad-relación, por ejemplo, utilizan una representación mediante un tipo de grafos que indica la relación entre componentes.

- La propagación de un virus informático en una red también puede ser modelizada mediante grafos.

En general, la resolución de problemas relacionados con la computación deriva, tarde o temprano, en la implementación de un algoritmo. Desde este punto de vista, resulta importante conocer la complejidad del algoritmo candidato a solución, pues interesa saber la cantidad de recursos que necesitará en cuanto a tiempo y almacenamiento.

Por lo anteriormente mencionado, se puede afirmar que la teoría de grafos es de interés para las Ciencias de la Computación.

En consecuencia, el estudio teórico de una clase de grafos interesante puede proporcionar resultados de aplicación directa o indirecta en el ámbito computacional.

El estudio abordado en esta memoria se centra en el contexto de los digrafos, o grafos dirigidos, en los cuales sus vértices o nodos pueden conectarse mediante arcos dirigidos. Por contraposición, en los grafos no dirigidos, las aristas entre vértices representan una conexión bidireccional. En este sentido, los grafos no dirigidos pueden contemplarse como el subconjunto de los digrafos simétricos, considerando cada arista como la unión de dos arcos de orientaciones opuestas. Los digrafos fuertemente conexos minimales o simplemente $M S D$, por sus siglas en inglés (minimal strong digraphs), son una clase muy interesante de digrafos, pues su definición considera apenas dos condiciones necesarias. La primera es la propiedad de la conexión fuerte, esto es, dados dos vértices cualesquiera, existe un camino dirigido que permite conectar uno con otro. La segunda es la propiedad de minimalidad, lo que implica que el digrafo no puede contener arcos innecesarios para la coenxión fuerte.

Lacalle, Marijuán y Pozo [7], en 2018, establecen analogías interesantes entre los MSD y una clase muy importante de grafos: los árboles. En particular, un árbol lineal contiene exactamente dos hojas en sus extremos; un MSD, bajo una estructura lineal, contiene ciclos de longitud corta y vértices de grado bajo y mantiene una propiedad similar a la de los árboles lineales, pues contiene exactamente 
dos vértices lineales en sus extremos. Parecería natural, pues, intentar establecer una cota inferior para el número de vértices lineales contenidos en un MSD, en función de la longitud de un ciclo, así como también en función del grado de los vértices del MSD.

En ese mismo trabajo, y usando la descomposición de un digrafo SC orejas, se comprueba que un MSD produce un árbol generador dirigido y un bosque de árboles con raíz invertidos. Puesto que este proceso parte de la construcción de un ciclo inicial, sería interesante estudiar estas orejas, pues podrían proporcionar información adicional sobre la estructura cíclica del MSD.

Además, resulta interesante el estudio del problema espectral de los digrafos ya que, mientras los digrafos fuertemente conexos pueden ser representados por matrices de adyacencia irreducibles, los MSD pueden representarse mediante matrices de adyacencia casi irreducibles. Para el caso de las matrices reducibles que representan a digrafos que no son fuertemente conexos, el problema espectral se reduce de manera automática a sus componentes irreducibles. El artículo [7] presenta una acotación de los coeficientes del polinomio característico para árboles, que se conjetura cierta para los MSD a partir del análisis de todos los MSD que se pueden generar hasta con quince vértices (el procedimiento para generar todos los MSD fue descrito por Lacalle y Marijuán en 2012: [6, 41]). Parece entonces razonable intentar avanzar en la prueba de la conjetura. Este aspecto resulta de interés, puesto que el espectro de un digrafo es un factor invariante bajo isomorfismo.

Para ello, utilizando el teorema de los coeficientes, podemos investigar la estructura de los ciclos de un MSD. En particular, dado un ciclo, ¿cómo se relaciona con el resto de ciclos y vértices del MSD? ¿Cómo afecta la eliminación de sus arcos a las propiedades de conexión del resto del MSD?

Finalmente, si establecemos propiedades que aclaran las posibles estructuras cíclicas y/o acotan el número de vértices lineales, se podría abordar el problema de complejidad algorítmica, por ejemplo, para la búsqueda de ciclos o caminos dirigidos de longitud maximal; para lo cual se podría partir de dos intuiciones aparentemente contrapuestas: por un lado, conociendo que la búsqueda de un subdigrafo generador minimal fuertemente conexo es un problema NP-duro; y, por otro lado, de que la cantidad de MSDs que se pueden generar con determinado número de vértices no es muy grande en comparación con la cantidad de otras clases de digrafos. 
Las definiciones de los términos mencionados en esta sección se encuentran en el Capítulo 2.

\subsection{Objetivos}

Los objetivos que esta tesis persigue se listan a continuación:

- Estudiar la manera en la que un ciclo puede estar contenido en un MSD, es decir, las distintas formas en las que un ciclo contenido en un MSD puede conectarse con el resto del digrafo, y determinar, en la medida de lo posible, la estructura de los ciclos de un MSD.

- Estudiar la consecuencia del objetivo anterior en tértminos de cotas inferiores para el número de vértices lineales de un MSD, en función de la longitud de sus ciclos.

- Estudiar si la existencia de vértices con un grado alto de entrada o de salida implica también una cota inferior del número de vértices lineales contenidos en un MSD.

- Utilizar los resultados sobre la estructura de ciclos con el fin de buscar condiciones necesarias para que una familia de números reales sean los coeficientes del polinomio característico de un MSD.

- Utilizar, si es posible, los resultados de acotación del número de vértices lineales para proponer algoritmos eficientes de búsqueda de ciclos de longitud máxima contenidos en un MSD. 


\section{Capítulo 2}

\section{Introducción teórica}

\subsection{Nociones básicas. Notación}

En este capítulo introduciremos los conceptos y nociones básicas que el lector debe conocer y tener presente para la comprensión de este trabajo. Adicionalmente se fijará la notación a utilizar. Para el desarrollo de esta sección teórica se han consultado las fuentes [1-3, 6, 7, 9-11, 14, 18, 30-33], cuyos resultados han sido citados textualmente en algunos casos y adaptados en otros.

De manera general, los grafos pueden ser entendidos como una estructura conformada por vértices y aristas que relacionan estos vértices entre sí. Formalmente: un grafo $G$ es un par $=(V, E)$ donde $V$ es un conjunto finito no vacío cuyos elementos llamaremos vértices (o nodos) y $E$ es un conjunto cuyos elementos son subconjuntos de $V$ de cardinal uno o dos. Los de cardinal uno se llaman lazos y los de cardinal dos se llaman aristas. Se dice que una arista $\{x, y\}$ conecta los vértices $x$ e $y$ y, también, que es incidente con ellos. También usaremos $x y$ para denotar a la arista $\{x, y\}$.

El orden de un grafo es el número de vértices en $V$, y normalmente lo denotaremos por $n$. El tamaño de un grafo es el número de aristas en $E$, y normalmente lo denotaremos por $m$. El grado de un vértice $v_{i} \in V$ (para todo $1 \leq i \leq n$ ) se denota como $d\left(v_{i}\right)$ y es el número de aristas incidentes con $v_{i}$.

La figura 2.1 representa un ejemplo de grafo; se puede apreciar que, por ejemplo, $d\left(v_{2}\right)=3$. Cada lazo $\{x\}$ aporta 2 al grado del vértice $x$, de modo que $d\left(v_{18}\right)=4$. Nótese que en todo grafo $m=\frac{\sum_{v \in V} d(v)}{2}$.

Un camino en un grado es una sucesión de vértices distintos $p=v_{1} v_{2} \cdots v_{q}$, $q \geq 2$, tal que $v_{i} v_{i+1}$ es una arista para todo $1 \leq i \leq q-1$. En este caso se dice 


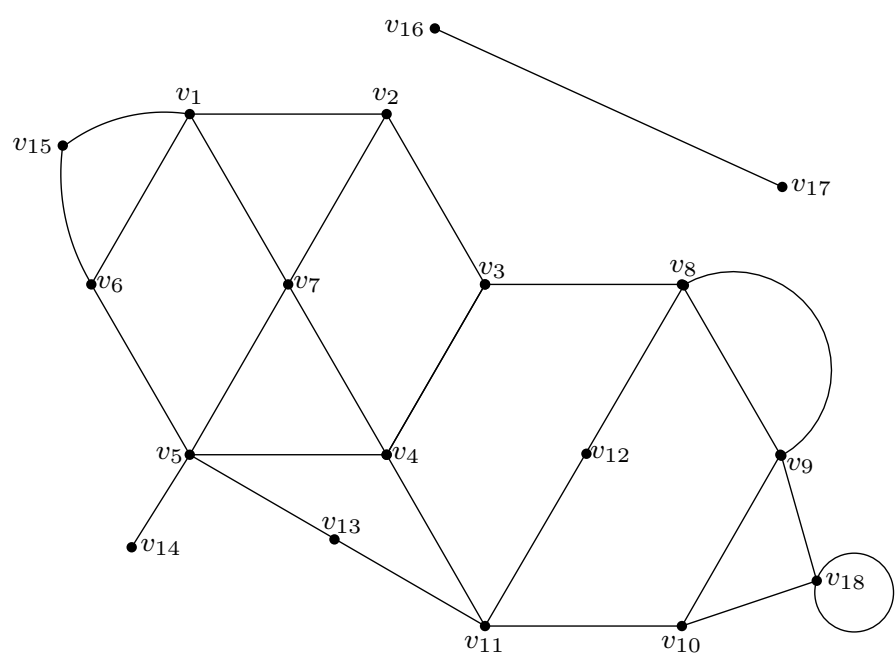

Figura 2.1: Ejemplo de un grafo.

que los vértices $v_{1}$ y $v_{p}$ están conectados por el camino $p$. Se dice que un grafo es conexo si todo par de vértices está conectado por un camino.

En un grafo no conexo existen dos o más subconjuntos de $V$ de modo que no es posible encontrar ningún camino entre vértices de subconjuntos distintos. En el grafo de la figura 2.1 se puede apreciar que existe un subconjunto $V_{1} \subset V$, donde $V_{1}=\left\{v_{16}, v_{17}\right\}$, cuyos elementos no pueden conectarse mediante camino alguno con los vértices de $V-V_{1}$. Por tanto, el grafo de la figura no es conexo.

Un grafo ponderado es una terna $(V, E, w)$ donde $(V, E)$ es un grafo y $w: E \rightarrow$ $\mathbb{R}$ es una función peso sobre el conjunto de aristas. Al número $w(x y)$ se le llama peso de la arista $x y$. El grafo de la figura 2.2 es un ejemplo de grafo ponderado y conexo en donde, por ejemplo, el peso de la arista $v_{5} v_{7}$ es de 8 .

En un grafo, la relación que una arista $u v$ establece entre los dos vértices $u \mathrm{y}$ $v$ es simétrica. La rotura de esta simetría para introducir una orientación en esa relación nos lleva al concepto de grafo dirigido. Un grafo dirigido o digrafo $D$ es un par $D=(V, A)$ donde $V$ es un conjunto finito no vacío y $A$ es un subconjunto del producto cartesiano $V \times V$. Llamaremos vértices a los elementos de $V$ y arcos a los de $A$. A los arcos de la forma $(v, v)$ los llamaremos lazos. Si $(u, v \in A$, diremos que $u$ es adyacente $a v$, que $v$ es adyacente desde $u$, que el arco $(u, v)$ es incidente en los vértices $u$ y $v$, que $(u, v)$ une o conecta el vértice $u$ con el vértice $v$ y que $u$ y $v$ son, respectivamente, el vértice inicial y el vértice final del arco $(u, v)$. También usaremos la notación $u v$ para referirnos al arco $(u, v)$. 


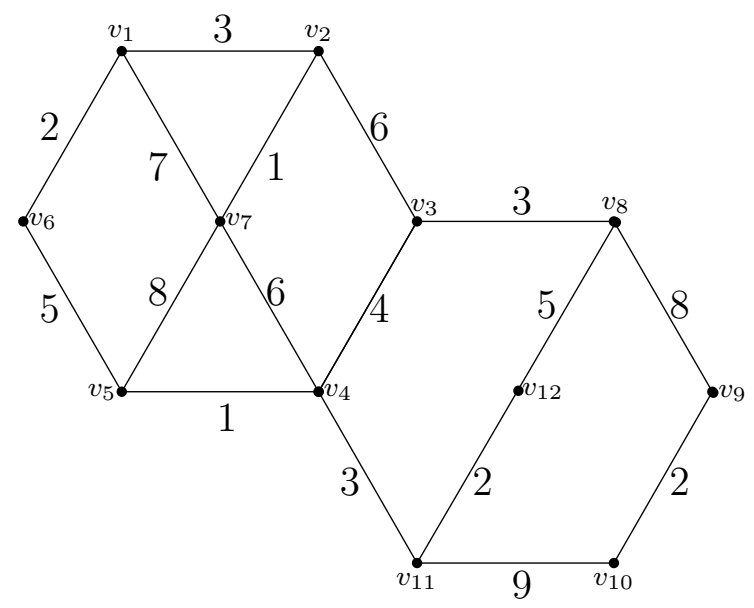

Figura 2.2: Ejemplo de un grafo conexo y ponderado.

De manera similar a los grafos, el orden de un digrafo es el número $|V|$ de vértices y lo denotaremos por $n$. El tamaño de un digrafo es el número $|A|$ de arcos y lo denotaremos por $m$.

El grado de salida (o exgrado) de un vértice $v \in V$ es igual al número de arcos que tienen a $v$ como vértice inicial y se denota como $d^{+}(v)$. El grado de entrada (o ingrado) de un vértice $v \in V$ es igual al número de arcos que tienen a $v$ como vértice final y se denota como $d^{-}(v)$. Nótese que en un digrafo se verifica que $\sum_{v \in V} d^{+}(v)=\sum_{v \in V} d^{-}(v)=m$.

Un camino dirigido es una secuencia de vértices distintos $v_{1}, v_{2}, \ldots, v_{q}, q \geq 2$, tal que $v_{i} v_{i+1}$ es un arco para $i=1,2, \ldots, q-1$. Un camino desde un vértice $u$ hasta un vértice $v$ lo denotamos como un uv-camino. Un ciclo de longitud $q$ o un $q$-ciclo es un camino $v_{1}, v_{2}, \ldots, v_{q}$ cerrado por el arco $v_{q} v_{1}$ y lo denotamos como $C_{q}$. En esta memoria consideraremos solamente caminos y ciclos dirigidos.

Un digrafo conexo es un digrafo cuyo grafo subyacente (el que se obtiene al sustituir todos los arcos por aristas) es conexo. Decimos que un digrafo $D=(V, A)$ es fuertemente conexo si para todo $u, v \in V$ existe un $u v$-camino.

En un digrafo fuertemente conexo (que denotaremos abreviadamente por $S D$, de sus siglas en inglés Strong Digraph) se verifica que $d^{+}\left(v_{i}\right) \geq 1$ y $d^{-}\left(v_{i}\right) \geq 1$ para todo $v_{i} \in V$. Diremos que $v_{i}$ es un vértice lineal si satisface que $d^{-}\left(v_{i}\right)=d^{+}\left(v_{i}\right)=$ 1. En el ejemplo de la figura 2.3 se representa un digrafo que no es fuertemente conexo pues, por ejemplo, el vértice $v_{7}$ tiene únicamente arcos de salida y no es 
posible encontrar un camino que llegue hasta $v_{7}$ desde ningún vértice; tampoco es posible encontrar un $v_{1} v_{5}$-camino en el digrafo. Además, los vértices $v_{14} \mathrm{y} v_{17}$ tienen únicamente arco de entrada y no es posible encontrar un camino que con origen en estos vértices. En esta figura se pueden encontrar más ejemplos que evidencian la carencia de la propiedad de conexión fuerte. En la figura 2.4 se muestra un digrafo que tiene la propiedad de ser fuertemente conexo. Nótese que un SD es un digrafo conexo, sin embargo un digrafo conexo no es necesariamente fuertemente conexo.

Sea $D=(V, A)$ un digrafo. Decimos que el digrafo $D^{\prime}=\left(V^{\prime}, A^{\prime}\right)$ es un subdigrafo de $D$ si $V^{\prime} \subset V$ y $A^{\prime} \subset A$. Un subdigrafo $D^{\prime}=\left(V^{\prime}, A^{\prime}\right)$ es inducido si $A^{\prime}$ está conformado por todos los arcos de $A$ que unen vértices de $V^{\prime}$.

La definición de digrafo ponderado se puede deducir de manera análoga a partir del concepto de grafo ponderado. No obstante, en esta memoria nos centraremos en digrafos conexos y no ponderados.

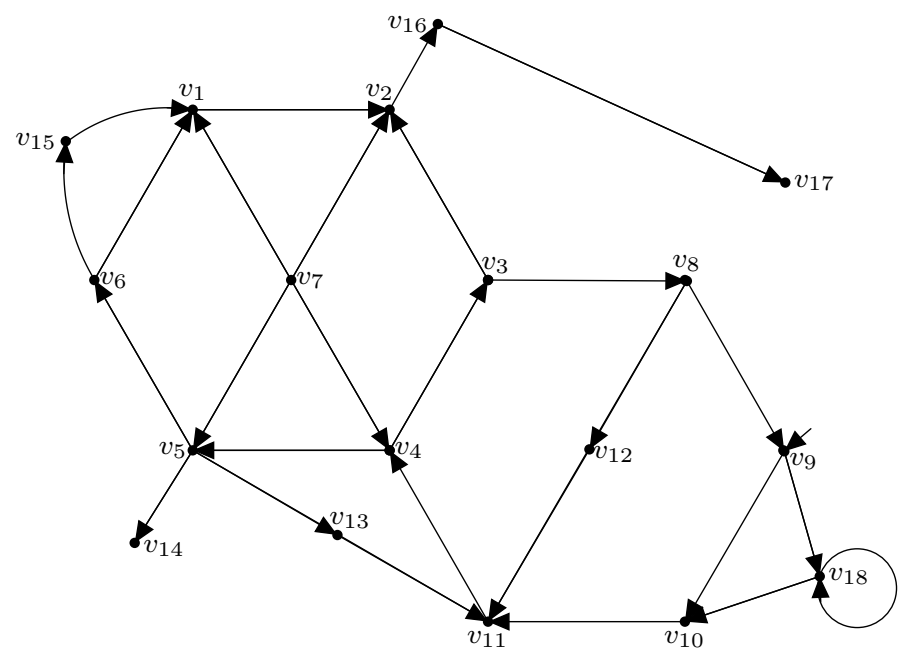

Figura 2.3: Ejemplo de un digrafo conexo.

Un arco $v_{i} v_{j}$ en un digrafo $D$ es transitivo si existe un $v_{i} v_{j}$-camino distinto del arco $v_{i} v_{j}$. Si un $v_{i} v_{j}$-camino y un arco $v_{i} v_{j}$ son distintos, la unión del $v_{i} v_{j}$-camino y el arco $v_{i} v_{j}$ recibe el nombre de pseudociclo. En el ejemplo de la figura 2.3 existen dos $v_{6} v_{1}$-caminos: uno de ellos es $v_{6}, v_{15}, v_{1}$ y el otro es simplemente el arco $v_{6} v_{1}$. Por lo tanto, este arco es transitivo. En este ejemplo se pueden encontrar más casos de arcos transitivos. En la figura 2.4 se muestra un digrafo que no contiene arcos transitivos. 
Definimos digrafo fuertemente conexo minimal ( $M S D$ por sus siglas en inglés) como aquel SD que pierde la propiedad de conexión fuerte al eliminar cualquiera de sus arcos. Geller[10] y Hedetniemi [11] demostraron que si $D$ es un digrafo fuertemente conexo, entonces $D$ es minimal si y solo si $D$ no contiene arcos transitivos o, equivalentemente, si y solo si $D$ no contiene pseudociclos. Los lazos se consideran arcos transitivos, pues son redundantes a efectos de conexión fuerte.

En la figura 2.4 se muestra un ejemplo de MSD conformado por 18 vértices.

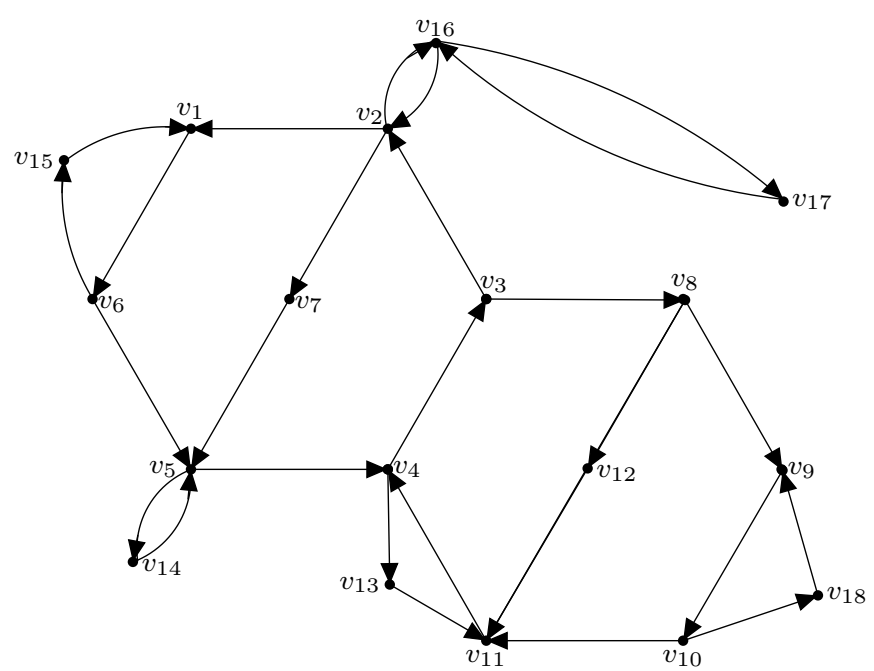

Figura 2.4: Ejemplo de un MSD de orden $n=18$.

Dados dos digrafos $D_{1}=\left(V_{1}, A_{1}\right)$ y $D_{2}=\left(V_{2}, A_{2}\right)$, decimos que $D_{1}$ y $D_{2}$ son isomorfos si existe una biyección $f: V_{1} \rightarrow V_{2}$ tal que para todo par de vértices $u, v \in V_{1}$, los vértices $u^{\prime}=f(u)$ y $v^{\prime}=f(v)$ son adyacentes en $D_{2}$ si y solo si $u$ y $b$ son adyacentes en $D_{1}$. En la figura 2.5 se pueden apreciar dos digrafos isomorfos.

Tanto un grafo no dirigido como un digrafo pueden ser representados por una matriz cuadrada de dimensiones $n \times n$ (donde $n=|V|$ ) cuyos elementos pueden tener valores únicamente binarios (0 ó 1$)$. A esta matriz se le conoce como matriz de adyacencia del digrafo $D$ (o del grafo no dirigido $G$ ) y la denotamos como $W_{D}$ (o $W_{G}$ respectivamente). Esta matriz resulta de numerar los vértices de $D$ (o $G$ ) en orden secuencial ascendente: $v_{1}, v_{2}, \ldots, v_{n}$. Entonces, con respecto a esta numeración, en la matriz de adyacencia el elemento $w_{i j}$ cumple la siguiente condición: 

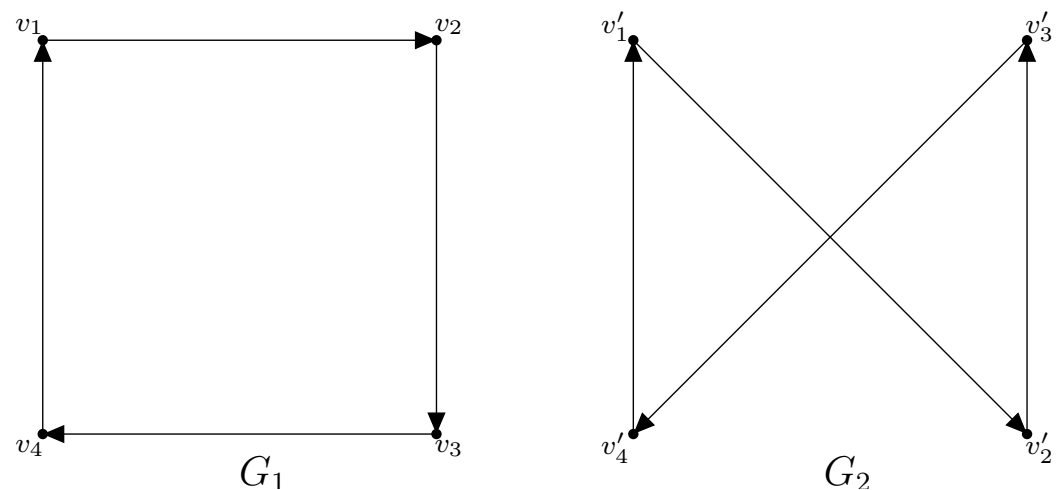

Figura 2.5: Ilustración de dos MSD isomorfos.

$$
w_{i j}= \begin{cases}1 & \text { si en } D \text { existe el } \operatorname{arco} v_{i} v_{j} \\ 0 & \text { si en } D \text { no existe el arco } v_{i} v_{j}\end{cases}
$$

De manera general la matriz de adyacencia $W$ tiene la siguiente forma:

$$
W=\left[\begin{array}{cccc}
w_{11} & w_{12} & \ldots & w_{1 n} \\
w_{21} & w_{22} & \ldots & w_{2 n} \\
\vdots & \vdots & \ddots & \vdots \\
w_{n 1} & w_{n 2} & \ldots & w_{n n}
\end{array}\right]
$$

En los grafos no dirigidos, la matriz de adyacencia $W_{G}$ de un grafo $G$ es simétrica, es decir, $w_{i j}=w_{j i}$ para todo $i, j$ tal que $1 \leq i, j \leq n$. Esto se debe a que sus aristas no son dirigidas y son bidireccionales, por lo que la existencia de la arista $v_{i} v_{j}$ implica también la existencia de la arista $v_{j} v_{i}$. Dado que, generalmente, se consideran solo grafos no dirigidos sin lazos, entonces los elementos de la diagonal principal de la matriz de adyacencia son 0 , esto es $w_{i i}=0$ para todo $i$, tal como se ilustra a continuación:

$$
W=\left[\begin{array}{cccc}
0 & w_{12} & \ldots & w_{1 n} \\
w_{21} & 0 & \ldots & w_{2 n} \\
\vdots & \vdots & \ddots & \vdots \\
w_{n 1} & w_{n 2} & \ldots & 0
\end{array}\right]
$$

Por ejemplo, sea $G$ el grafo no dirigido de la figura 2.6. La matriz de adyacencia $M_{G}$ sería la siguiente (nótese que se cumple la propiedad de simetría). 


$$
M_{G}=\left[\begin{array}{lllllll}
0 & 1 & 0 & 0 & 0 & 1 & 0 \\
1 & 0 & 1 & 0 & 0 & 0 & 1 \\
0 & 1 & 0 & 1 & 0 & 0 & 0 \\
0 & 0 & 1 & 0 & 1 & 0 & 0 \\
0 & 0 & 0 & 1 & 0 & 1 & 1 \\
1 & 0 & 0 & 0 & 1 & 0 & 0 \\
0 & 1 & 0 & 0 & 1 & 0 & 0
\end{array}\right]
$$

En el caso de un MSD $D$, su matriz de adyacencia $W_{D}$ no es necesariamente simétrica, pues la existencia de un arco $v_{i} v_{j}$ no necesariamente implica la existencia del arco $v_{j} v_{i}$. Sin embargo, los elementos de la diagonal principal de $W_{D}$ siempre tendrán el valor 0 (es decir, $w_{i i}$ para todo $i$ ) pues, como se ha dicho anteriormente, un MSD no puede contener lazos.

Por ejemplo, sea $D$ el MSD de la figura 2.7. La matriz de adyacencia $M_{D}$ sería la siguiente (nótese que esta matriz no cumple la propiedad de simetría).

$$
M_{D}=\left[\begin{array}{lllllll}
0 & 0 & 0 & 0 & 0 & 1 & 0 \\
1 & 0 & 0 & 0 & 0 & 0 & 1 \\
0 & 1 & 0 & 0 & 0 & 0 & 0 \\
0 & 0 & 1 & 0 & 0 & 0 & 0 \\
0 & 0 & 0 & 1 & 0 & 0 & 0 \\
0 & 0 & 0 & 0 & 1 & 0 & 0 \\
0 & 0 & 0 & 0 & 1 & 0 & 0
\end{array}\right]
$$

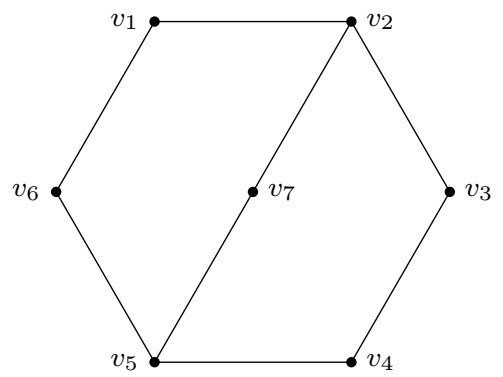

Figura 2.6: Ejemplo de un grafo no dirigido de orden $n=7$.

Toda matriz de adyacencia tiene la particularidad de ser no negativa, es decir $w_{i j} \geq 0$ para todo $1 \leq i, j \leq n$. 


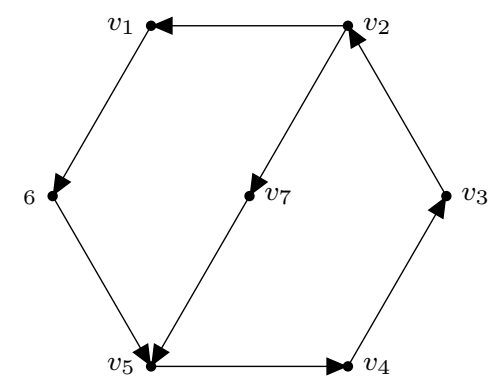

Figura 2.7: Ejemplo de un MSD de orden $n=7$.

Una matriz no negativa $M$ de dimensiones $n \times n$ es reducible si existe una matriz de permutación $P$ tal que

$$
P M P^{T}=\left[\begin{array}{cc}
X_{11} & 0 \\
X_{21} & X_{22}
\end{array}\right]
$$

en donde $X_{11}$ y $X_{22}$ son matrices cuadradas. En caso contrario $M$ es irreducible $[31,32]$. Es decir, la matriz $M$ es irreducible si y solo si es la matriz de adyacencia de un digrafo fuertemente conexo [9]. Por lo tanto, la matriz de adyacencia de un MSD es irreducible. Pero, si cambiamos en dicha matriz cualquier entrada igual a 1 por un 0 , la matriz pasa a ser reducible; una matriz con dicha propiedad se llama casi reducible.

Por ejemplo, consideremos los digrafos de la figura 2.1 en la que el digrafo $D_{2}$ es fuertemente conexo (además es minimal), mientras que el digrafo $D_{1}$ no lo es.
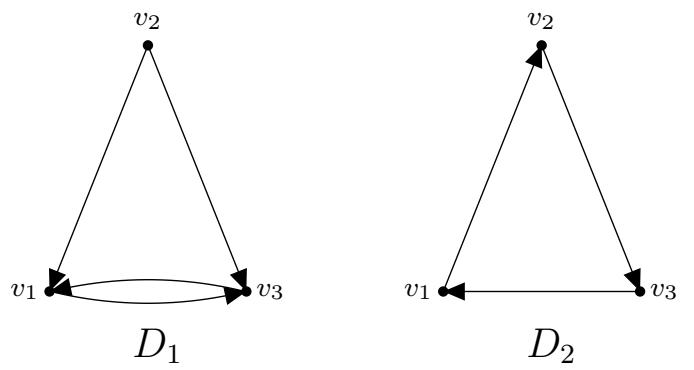

Figura 2.8: Ilustración de un digrafo conexo no fuertemente conexo $D_{1}$ y de un digrafo fuertemente conexo $D_{2}$.

Las matrices de adyacencia de los digrafos de la figura anterior se muestran a continuación. Nótese que la reducibilidad de $M_{1}$ no es evidente a partir de su 
expresión.

$$
M_{1}=\left[\begin{array}{lll}
0 & 0 & 1 \\
1 & 0 & 1 \\
1 & 0 & 0
\end{array}\right] \quad \text { y } M_{2}=\left[\begin{array}{lll}
0 & 1 & 0 \\
0 & 0 & 1 \\
1 & 0 & 0
\end{array}\right]
$$

Las matrices de adyacencia de los ejemplos de la figura 2.1 tienen dimensión $3 \times 3$. Por lo tanto existen seis permutaciones posibles para cada una de ellas, con matrices de permutación

$$
\begin{aligned}
& P_{1}=\left[\begin{array}{lll}
1 & 0 & 0 \\
0 & 1 & 0 \\
0 & 0 & 1
\end{array}\right] P_{2}=\left[\begin{array}{lll}
0 & 1 & 0 \\
1 & 0 & 0 \\
0 & 0 & 1
\end{array}\right] P_{3}=\left[\begin{array}{lll}
0 & 0 & 1 \\
1 & 0 & 0 \\
0 & 1 & 0
\end{array}\right] \\
& P_{4}=\left[\begin{array}{lll}
0 & 1 & 0 \\
0 & 0 & 1 \\
1 & 0 & 0
\end{array}\right] P_{5}=\left[\begin{array}{lll}
0 & 0 & 1 \\
0 & 1 & 0 \\
1 & 0 & 0
\end{array}\right] P_{6}=\left[\begin{array}{lll}
1 & 0 & 0 \\
0 & 0 & 1 \\
0 & 1 & 0
\end{array}\right]
\end{aligned}
$$

Es inmediato comprobar, entonces, que

$$
P_{3} M_{1} P_{3}^{T}=\left[\begin{array}{lll}
0 & 1 & 0 \\
1 & 0 & 0 \\
1 & 1 & 0
\end{array}\right]
$$

permitiendo determinar, directamente a partir de la definión, que la matriz de adyacencia del digrafo $D_{1}$ es reducible. Sin embargo, para $M_{2}$ no existe permutación alguna que produzca tal resultado. Evidentemente, el hecho de que el digrafo $D_{1}$ de la Figura no sea fuertemente conexo, mientras que el digrafo $D_{2}$ sí lo es, nos permite alcanzar las mismas conclusiones de una forma más directa.

Las siguientes definiciones concernientes a los MSD se encuentran descritas en $[1,4]$. La contracción de un subdigrafo consiste en la reducción del subdigrafo a un único vértice. La contracción de un subdigrafo fuertemente conexo, contenido en un MSD, produce otro MSD.

Un vértice $v$ en un digrafo es llamado punto de corte si la eliminación de $v \mathrm{y}$ todos sus arcos incidentes produce un digrafo no conexo. Llamamos componente fuertemente conexa (o $S C$, por las siglas en inglés de Strong Component) a un subdigrafo fuertemente conexo maximal, es decir, un subdigrafo al que no se le pueden añadir arcos y/o vértices manteniendo la conexión fuerte.

Llamamos árbol no dirigido (o simplemente árbol) a un grafo conexo y sin ci- 
clos. Los árboles también pueden caracterizarse como grafos conexos para los que $m=n-1$ o como grafos conexos minimales. Es decir, un árbol tiene la propiedad de minimalidad como grafo conexo: si en un árbol $T$ se elimina cualquiera de sus aristas, entonces $T$ pierde la propiedad de conexión. Un árbol dirigido doble $\overleftrightarrow{T}$ puede ser obtenido a partir de un árbol no dirigido $T$ mediante la sustitución de cada arista $u v$ de $T$ por dos arcos $u v, v u$ de $\overleftrightarrow{T}$ (ver ejemplo en figura 2.9). Nótese que $\overleftrightarrow{T}$ es fuertemente conexo y es minimal y por lo tanto $\overleftrightarrow{T}$ es un MSD Es decir, la clase de los árboles dobles es un subconjunto de los MSD.
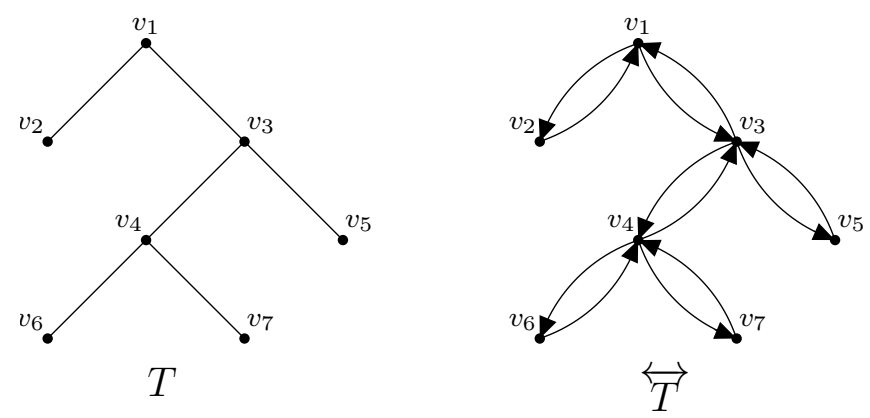

Figura 2.9: Ilustración de un árbol no dirigido $T$ y de un árbol dirigido doble $\overleftrightarrow{T}$ obtenido a partir de $T$.

En un SD, cada arco está contenido en un ciclo. En efecto, dado el arco uv, se obtiene un ciclo al añadir a dicho arco el vu-camino que debe existir por conexión fuerte. En consecuencia, en todo digrafo fuertemente conexo es posible encontrar al menos un ciclo.

La descomposición en orejas $[3,7]$ de un digrafo fuertemente conexo $D=$ $(V, A)$ es una una sucesión de digrafos $P_{0}, P_{1}, \ldots, P_{k}$, en donde $P_{0}=\left(V_{0}, A_{0}\right)$ es un ciclo y cada $P_{j}=\left(V_{j}, A_{j}\right)$, para $1 \leq j \leq k$, es un camino o un ciclo que cumple con las siguientes propiedades:

a) $P_{j}$ y $P_{j^{\prime}}$ son arco-disjuntos si $j \neq j^{\prime}$.

b) Para cada $j=1, \ldots, k$ : si $P_{j}$ es un ciclo, entonces tiene solamente un vértice común con $\bigcup_{j^{\prime}=0}^{j-1} V_{j^{\prime}}$. Si no, los vértices terminales de $P_{j}$ son vértices distintos de $\bigcup_{j^{\prime}=0}^{j-1} V_{j^{\prime}}$ y ningún otro vértice de $P_{j}$ pertenece a $\bigcup_{j^{\prime}=0}^{j-1} V_{j^{\prime}}$.

c) $\bigcup_{j=0}^{k} A_{j}=A$.

Llamamos oreja (o asa) a cada $P_{j}$. Los MSD satisfacen la condición de que cada oreja tiene al menos un vértice nuevo y dos arcos nuevos. Denotamos co- 
mo $v_{i}^{j}$ a cada vértice $v_{i}$ que pertenece a la oreja $P_{j}$. A manera de ejemplo, se muestra la construcción paso a paso del MSD de la figura 2.4, al que llamaremos $D$, para lo cual se utilizará una adaptación del proceso descrito en $[7$, Teorema 20]: Primeramente escogemos un ciclo cualquiera en $D$, por ejemplo $P_{0}=v_{1}^{0}, v_{6}^{0}, v_{5}^{0}, v_{4}^{0}, v_{3}^{0}, v_{2}^{0}, v_{1}^{0}$ que será la primera oreja (figura 2.10). Nótese que este ciclo tiene longitud $k_{0}=6$.

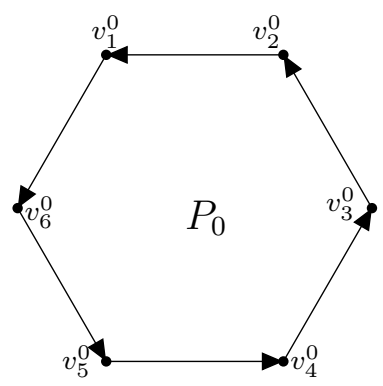

Figura 2.10: Construción de la primera oreja $\left(P_{0}\right)$ de un MSD.

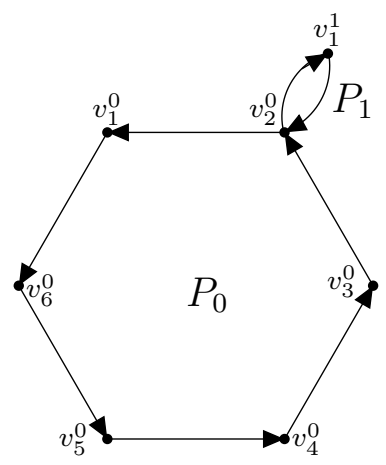

Figura 2.11: Construción de la oreja $P_{1}$.

Luego, para cada vértice $v_{i}^{0} \in P_{0}$ tal que $d^{+}\left(v_{i}^{0}\right)>1$ y $1 \leq i \leq k_{0}$, se construye el camino que inicia por el arco $v_{i}^{0} v_{j}^{1} \in D$, de tal manera que en algún momento se alcance nuevamente un vértice $v_{k_{0}^{\prime}}^{0} \in P_{0}$ sin haber pasado más de una vez por un mismo vértice. En el ejemplo de la figura $2.11 d^{+}\left(v_{2}^{0}\right)>1$, luego existe el arco $v_{2}^{0} v_{1}^{1}$, puesto que $v_{1}^{1} v_{2}^{0} \in D$, entonces $P_{1}=v_{2}^{0}, v_{1}^{1}, v_{2}^{0}$.

Este proceso se ejecuta de manera iterativa hasta construir todas las orejas posibles a partir de los arcos de salida de los vértices que pertenecen a $P_{0}$. Posteriormente se procede de la misma manera con los vértices de $\bigcup_{i \leq j} V_{i}$ hasta 
contruir todas las orejas que empiezan y terminan en vértices de dicho conjunto. En la figura 2.12 se puede apreciar la evolución de $D$ aplicando este proceso.
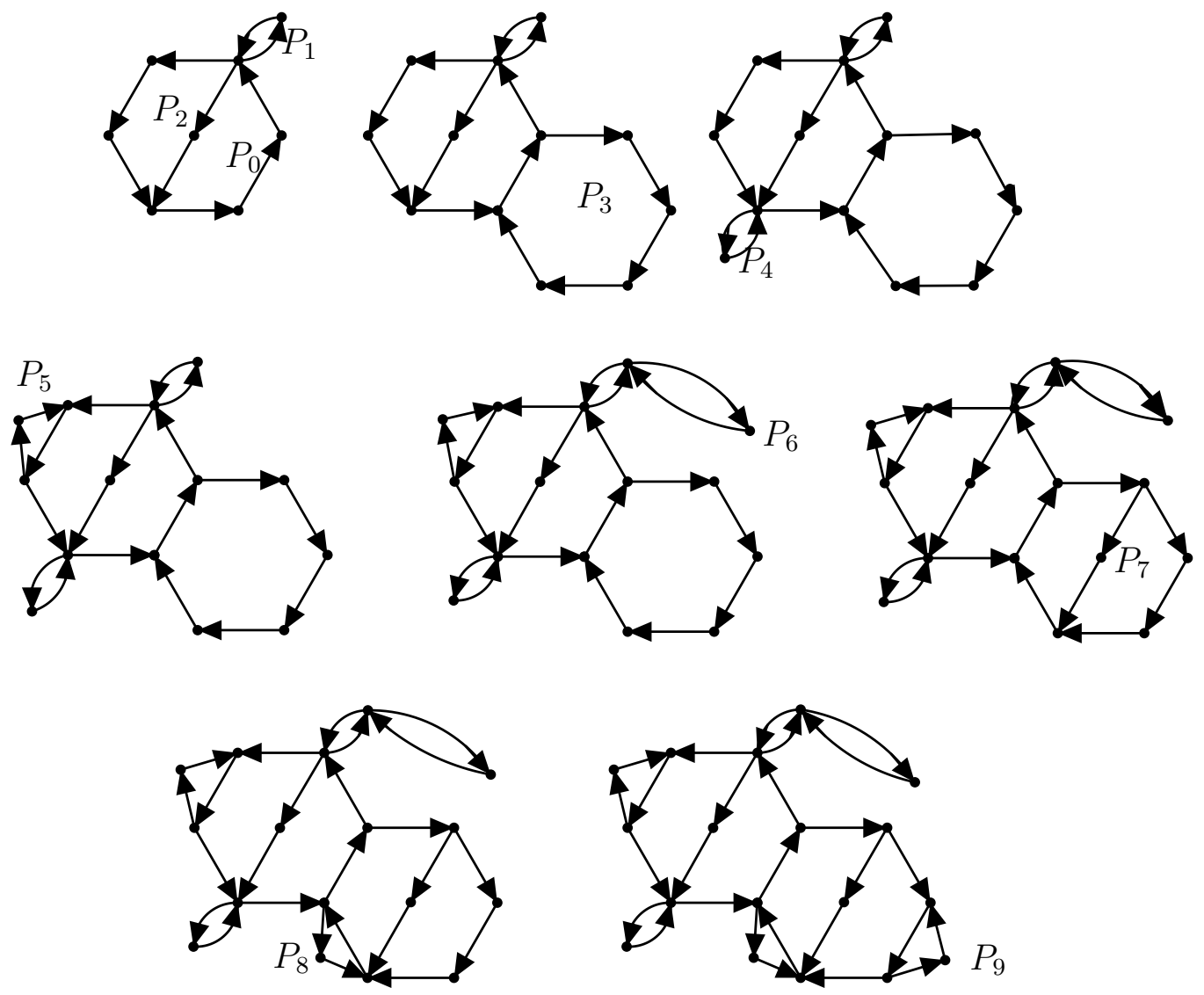

Figura 2.12: Construcción de un MSD mediante la adición de cada una de sus orejas.

Se denomina polinomio característico de un digrafo (o de un grafo no dirigido) al polinomio característico de su matriz de adyacencia, es decir $\chi_{D}(x)=\operatorname{det}(x$. $\left.I_{n}-M_{D}\right)$, que tiene la forma $x^{n}+k_{1} x^{n-1}+k_{2} x^{n-2}+\cdots+k_{n}$. El Teorema de los coeficientes [33] relaciona los coeficientes $k_{i}$ con las posibles formas de cubrir $i$ vértices de $D$ mediante ciclos disjuntos. Explícitamente,

$$
k_{i}=\sum_{L \in \mathcal{L}_{i}}(-1)^{p(L)}
$$

para $i=1, \ldots, n$, donde $\mathcal{L}_{i}$ es el conjunto de todos los subdigrafos lineales $L$ de $D$ con exactamente $i$ vértices (un subdigrafo de $D$ es lineal si es unión disjunta de ciclos) y $p(L)$ es el número de componentes de $L$ (es decir, el número de ciclos 
de los que se compone $L$ ). Es decir, para cada término $k_{i} x^{n-i}$ donde $1 \leq i \leq n$, $k_{i}$ es el número de maneras de cubrir $i$ vértices de $D$ mediante ciclos disjuntos, cada una contada con signo $(-1)^{c}$, siendo $c$ el número de ciclos.

Para el caso de los MSD puede haber a lo sumo una forma de cubrir todos sus vértices mediante ciclos disjuntos [7, Corolario 18]. Como consecuencia, en vista del Teorema de los coeficientes, si $D$ es un MSD, el término independiente $k_{n}$ satisface: $k_{n}=0$ si y solo si no existe un cubrimiento de todos los vértices de $D$ mediante ciclos disjuntos; $k_{n}=1$ si y solo si todos los vértices de $D$ son cubiertos con un número par de ciclos disjuntos; y, $k_{n}=-1$ si y solo si todos los vértices de $D$ son cubiertos con un número impar de ciclos disjuntos. Por lo tanto, si $k_{n} \neq 0$ entonces $k_{n}=(-1)^{c}$ en donde $c$ es el número de ciclos disjuntos con los que se cubren todos los vértices de $D$.

Por ejemplo, consideremos los MSD de la figura 2.13: en el caso (a) se puede apreciar que todos los vértices del digrafo pueden ser cubiertos mediante los ciclos disjuntos $C_{6}=v_{1}, v_{2}, v_{3}, v_{4}, v_{5}, v_{6}, v_{1}$ y $C_{2}=v_{7}, v_{8}, v_{7}$; en cambio, para el caso (b) no existe manera alguna de cubrir todos sus vértices mediante ciclos disjuntos. Para el ejemplo (a), dado que $n=8$, existe únicamente una manera de cubrir 8 vértices mediante ciclos disjuntos y dado que el número de ciclos disjuntos es par, entonces $k_{8}=(-1)^{2}=1$; además, existe solamente una manera de cubrir seis vértices mediante ciclos disjuntos (usando el ciclo $C_{6}$ ), entonces, como en este caso se utiliza únicamente un ciclo, $k_{6}=(-1)^{1}=-1$; y existen dos maneras de cubrir dos vértices, mediante el ciclo $v_{1}, v_{7}, v_{1}$ y mediante el ciclo $v_{7}, v_{8}, v_{7}$, entonces $k_{2}=(-1)^{1}+(-1)^{1}=-2$; mientras que $k_{1}=k_{3}=k_{4}=k_{5}=k_{7}=0$. Entonces, el polinomio característico del ejemplo dado en (a) es $x^{8}-2 x^{6}-x^{2}+1$. El polinomio característico del MSD dado en (b) es $x^{7}-x^{2}-x$.

\subsection{Estado del arte}

A continuación resumimos algunas propiedades concernientes a los MSD que han sido obtenidas como resultados de investigaciones previas a este trabajo. Haremos especial énfasis sobre las propiedades que usaremos posteriormente de forma explícita en algunas de nuestras demostraciones.

Gupta [8], en el año 1967, demostró la siguiente propiedad.

Lema 1. El tamaño de un $M S D D$ verifica que $n \leq m \leq 2(n-1)$. El tamaño de $D$ es $n$ si y solo si $D$ es un $n$-ciclo. El tamaño de $D$ es $2(n-1)$ si y solo si $D$ es 


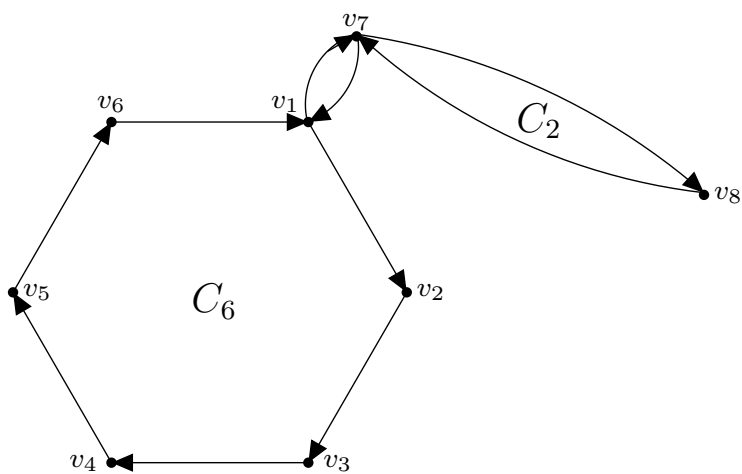

(a)

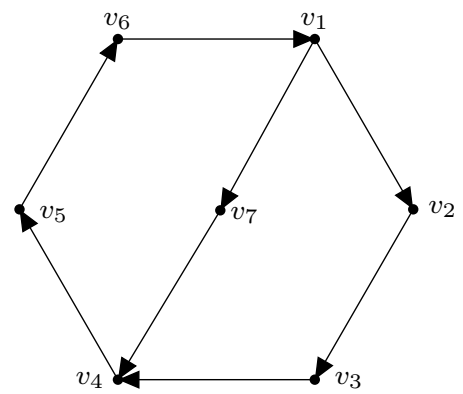

(b)

Figura 2.13: En el caso del MSD representado en (a) todos sus vértices pueden ser cubiertos mediante ciclos disjuntos. En el caso del MSD representado en (b) esto no es posible.

un árbol dirigido doble.

Posteriormente, en 1979, Brualdi y Hedrick [19] publicaron una demostración distinta y más completa para el Lema 1 en relación al resultado de Gupta. Además, los mismos autores caracterizaron los MSD de tamaño $2 n-3$ afirmando, entre otras propiedades, que este tipo de $\mathrm{MSD}$ contiene algún ciclo $C_{3}$ y ningún ciclo de longitud mayor a 3.

En 1991, Berge [4] demostró algunos resultados concernientes a los MSD. Explicitamos a continuación algunos de ellos. Dado un MSD y un subconjunto de sus vértices que genera un subdigrafo fuertemente conexo, la contracción de dicho subconjunto genera un digrafo fuertemente conexo, que también es minimal. Como corolario de esta propiedad, el autor enuncia el siguiente lema.

Lema 2. La contracción de un ciclo $C_{q}$ en un $M S D D$ conserva la minimalidad, esto es, produce otro MSD.

Según el Lema 2, si $D^{\prime}$ es el MSD resultante de la contracción de $C_{q}$ en $D$, entonces el orden del MSD $D^{\prime}$ será $n^{\prime}=n-q+1$ y su tamaño será $m^{\prime}=m-q$.

Además, Berge (y luego Brualdi y Ryser [9] en 1992) probaron que la siguiente propiedad se cumple para los MSD. Previamente, en 1968, fue demostrada de manera independiente por Dirac [12] y Plummer [13] en el contexto de los grafos 2-conexos.

Lema 3. Todo $M S D$ de orden $n \geq 2$ tiene al menos dos vértices lineales. 
En [10-15] se describen procedimientos que construyen grafos y digrafos extensos a partir de grafos y digrafos pequeños, respectivamente. Todos estos procedimientos añaden nuevos caminos entre dos vértices seleccionados previamente de una manera analítica. Cunningham [16] presentó una descomposición en árboles para MSDs, la cual fue caracterizada por Bhogadi [15] y utilizada para generar todos los MSDs de orden menor o igual que 12, así como todos los grafos denominados 2-conexos conformados por hasta 13 vértices. Un grafo 2-conexo es un grafo de orden mayor o igual a 2 en el que cada par de vértices pertenecen a algún ciclo no dirigido [12]. Sin embargo, estos procedimientos o bien no conservan la minimalidad del grafo o digrafo, o bien no son caracterizadas las condiciones bajo las cuales la minimalidad es preservada. Zhang y Guo [17], por su parte, diseñaron un precedimiento para enumerar todos los MSDs contruidos a partir de ciclos fundamentales de digrafos dados. Además caracterizaron las condiciones bajo las cuales se preserva la minimalidad [8].

En el año 2012, García-López y Marijuán [6] propusieron un algoritmo iterativo de construcción de MSD en relación a su orden. Para desarrollar esta tarea también demostraron algunas propiedades generales de los MSD. Por ejemplo: utilizando el Lema 2, presentaron una demostración más corta para el Lema 1, mediante inducción sobre el número de vértices $n$ del MSD. Presentaron también una versión simplificada de la demostración de Berge para el Lema 3, en la que también se usa inducción sobre $n$.

Los procesos fundamentales para crear un nuevo MSD a partir de uno existente, añadiendo un vértice, son las llamadas expansiones internas y externas. Definen los términos expansión interna y expansión externa como propiedades de los MSD: la expansión interna de un digrafo $D=(V, A)$ mediante el vértice $v \notin V$ sobre el arco $u w$ es el digrafo $i_{u w}(D)=\left(V \cup\{v\}, A^{*}\right)$ donde $A^{*}=A \cup\{u v, v w\}-$ $\{u w\}$. La expansión externa de $D=(V, A)$ mediante el vértice $v \notin V$ desde el vértice $u \in V$ hasta el vértice $w \in V$ es el digrafo $e_{u w}(D)=\left(V \cup\{v\}, A^{*}\right)$ donde $A^{*}=A \cup\{u v, v w\}$. Además si el vértice $w$ coincide con el vértice $u$ entonces $e_{u w}(D)=e_{u}(D)$ y se denomina expansión externa sobre el vértice $u$.

Sea $D=(V, A)$ un MSD y sean $u, w$ vértices tales que $u w \notin A$. La expansión externa $e_{u w}(D)$ de $D$ mediante el vértice $v \notin V$ desde el vértice $u$ hasta el vértice $w$ es un MSD si y solo si el digrafo $D+u w$ no contiene arcos transitivos distintos de $u w$. Sea $D^{*}=\left(V, A^{*}\right)$ un MSD de orden $n \geq 3$ y $v \in V$ un vértice lineal en $D^{*}$. Entonces, existe un MSD $D=(V-v, A)$ cuya expansión (interna o externa) 
mediante el vértice $v$ es el digrafo $D^{*}$.

Estas propiedades fueron útiles para el diseño e implementación de dos algoritmos. El primero de ellos calcula los MSD no etiquetados siguiendo un proceso de construcción mediante expasión interna y externa. La implementación de este algoritmo permitió calcular todos los MSD posibles hasta el orden 14 (y posteriormente los de orden 15 [41]), ampliando el resultado presentado por Bhogadi [15] en 1999. El segundo algoritmo implementado calcula las clases isospectrales de los MSD, determinando el digrafo y el polinomio característico de cada clase.

García-López, Marijuán y Pozo-Coronado [7] (2018) profundizan el estudio de los MSDs y demuestran varias propiedades nuevas, además de plantear una interesante analogía entre árboles y MSDs. Resumimos los principales resultados a continuación.

Los autores expresan que los árboles pueden ser vistos como grafos conexos con $n-1$ aristas; mientras que un MSD es un digrafo fuertemente conexo con $n \leq m \leq 2(n-1) \operatorname{arcos}$ (Lema 1). Además, las dos familias de grafos (con al menos dos vértices) satisfacen la propiedad de contener al menos dos vértices lineales (Lema 3). En el caso de los árboles, sus hojas pueden ser consideradas como vértices lineales.

Consideran que, en ambos casos, árboles y MSD, existen configuraciones con un máximo número de vértices lineales relacionados a su orden: los árboles estrella tienen $n-1$ y los ciclos dirigidos tienen $n$ vértices lineales. Consideran también que, en ambos casos, existen configuraciones con un vértice de grado máximo: aŕboles y MSD con forma de estrella.

Proponen el término MSD lineal y lo definen también por analogía con los árboles, puesto que existe un único árbol de orden $n$ con un número mínimo de vértices lineales: los grafos $L_{n}$.

Los MSD lineales tienen también configuraciónes lineales y se pueden construir de la siguiente manera.

Consideremos una árbol $L_{n}$ cualquiera, en donde los vértices $v_{1}$ y $v_{n}$ son lineales (como ejemplo iremos construyendo un MSD lineal a partir de un árbol lineal $L_{4}$, ver figura 2.14).

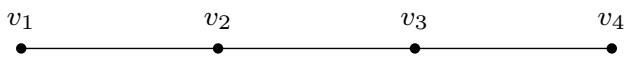

Figura 2.14: Ilustración de un ejemplo de árbol lineal $L_{4}$. 
Existen tres tipos básicos de MSD lineales, los cuales pueden ser nombrados como $D_{2}$ si el MSD es un ciclo de longitud 2; $D_{4}$ si el MSD tiene la configuración $C_{3} C_{3}$ donde los ciclos deben tener un arco en común en los dos vértices en los que incide; y $D_{2 n}$ si el MSD tiene la configuración $C_{3} C_{4} C_{4} \ldots C_{4} C_{3}$ y es de orden $2 n$ para $n \geq 3$ (ver ejemplo en figura 2.15).

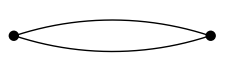

$D_{2}$

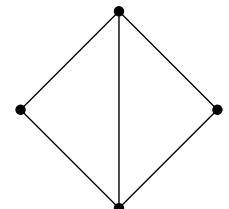

$D_{4}$

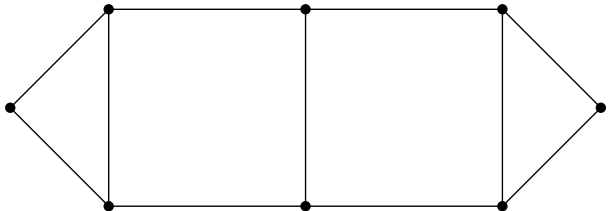

$D_{2 n}$

Figura 2.15: Ilustración de los tipos básicos de MSD lineales.

Ahora, en $L_{n}$ sustituimos cada arista $v_{i} v_{i+1}$ (para $1 \leq i \leq n-1$ ) por arcos dobles, lo que equivale a ciclos dirigidos $C_{2}$ o MSDs lineales $D_{2}$. Esto permite generar un MSD compuesto por una sucesión de ciclos $C_{2}$ que contiene un vértice lineal en cada uno de sus extremos (ver ejemplo en figura 2.16).

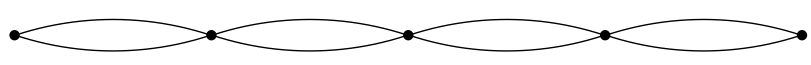

Figura 2.16: Ejemplo de MSD lineal formado por ciclos $C_{2}$.

Finalmente, todo ciclo $C_{2}$ puede ser reemplazado por cualquiera de los tipos básicos de MSD lineales. El digrafo resultante mantendrá un vértice lineal en cada uno de sus extremos (ver ejemplo en figura 2.17)

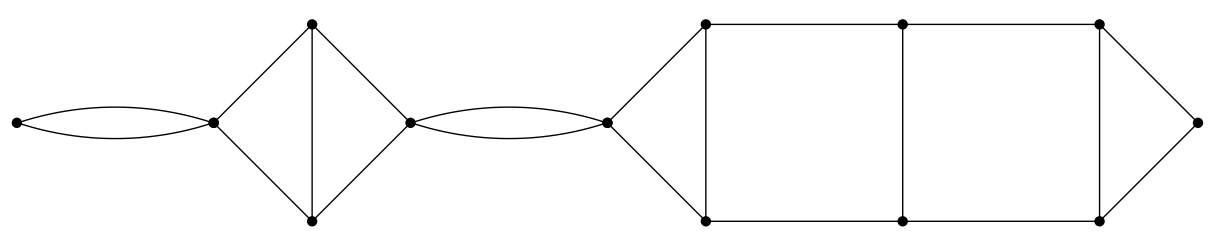

Figura 2.17: Ilustración de un ejemplo de MSD lineal.

A partir de esta construcción los autores presentan el siguiente lema y caracterización. 
Lema 4. En un MSD con exactamente dos vértices lineales, cada uno de ellos pertenece a un único ciclo. Además, estos ciclos son $C_{2} o C_{3}$.

Lema 5. Un MSD es lineal si y solo si contiene exactamente dos vértices lineales.

Adicionalmente, los autores afirman que si un MSD contiene un ciclo $C_{4}$ con un vértice lineal o un ciclo $C_{5}$, entonces contiene al menos tres vértices lineales.

En un árbol, cada vértice pertenece a un ciclo de longitud dos (considerando cada arista como un arco doble) y, por lo tanto, cualquier vértice es un vértice lineal o de corte. En un MSD lineal, cada vértice contenido en un ciclo $C_{2}$ es un vértice lineal o de corte.

En un árbol, toda arista es un corte. En el árbol dirigido doble, los arcos de cada $C_{2}$ constituyen un 2-corte. Entiéndase como 2-corte a un par de arcos de un digrafo fuertemente conexo que al ser eliminados lo convierte en un digrafo no conexo. La propiedad se generaliza a los MSD y así se cumple que cada ciclo de un MSD tiene un 2-corte.

Dados dos vértices $u$ y $v$ de cualquier árbol, existe un camino único que los conecta. Los autores generalizan este hecho como la propiedad camino-árbol para grafos y digrafos: para cada dos vértices $u$ y $v$ en un grafo (resp. digrafo) existe un único camino que los conecta (resp. un único camino dirigido desde $u$ hasta $v)$. Entonces, los árboles satisfacen la propiedad de camino-árbol, mientras que los MSD no. Si uv es un arco en un MSD entonces este camino es el único que los conecta, pero no se puede decir lo mismo si el arco $u v$ no existe en el MSD. Sin embargo, existe una subfamilia de la clase MSD en la que se mantiene la propiedad, los autores llaman a esta clase como digrafos de ciclos dirigidos.

Otra diferencia significativa entre árboles y MSD es la complejidad de los siguientes problemas algorítmicos: dado un grafo conexo ponderado y un digrafo fuertemente conexo ponderado, encontrar un árbol generador minimal y un subdigrafo generador minimal fuertemente conexo ( $M S T$ y $M S S S$ por sus siglas en inglés), respectivamente. Mientras existen varios algoritmos polinomiales para resolver el problema MST [34], el problema MSSS es NP-duro (ver [3]), inclusive si se trata de un digrafo no ponderado. Las definiciones referentes a la complejidad de algoritmos se encuentran más adelante en esta sección.

Cada árbol tiene como máximo un cubrimiento perfecto. Los autores prueban la siguiente propiedad análoga sobre los MSD.

Lema 6. Un MSD tiene como máximo un cubrimiento mediante ciclos disjuntos. 
El estudio realizado por los autores abarca el tema de los coeficientes del polinomio caracterśtico de los MSD. A continuación exponemos sus principales resultados.

Sea $T$ un árbol con $n \geq 2$ vértices, sea $\overleftrightarrow{T}=(V, A)$ un árbol dirigido doble obtenido a partir de $T$ mediante la sustitución de cada arista por arcos dirigidos dobles y sea $k_{m}(\overleftrightarrow{T})$ el coeficiente de $x^{n-m}$ en el polinomio característico mónico de la matriz de adyacencia de $\overleftrightarrow{T}$. Entonces,

1. $K_{m}(\overleftrightarrow{T})=0$ para todo $m$ impar tal que $1 \leq m \leq n$

2. $K_{m}(\overleftrightarrow{T}) \leq\left(\begin{array}{c}n-\frac{m}{2} \\ \frac{m}{2}\end{array}\right)$ para todo $m$ par tal que $2 \leq m \leq n$

3. $K_{m}(\overleftrightarrow{T})=\left(\begin{array}{c}n-\frac{m}{2} \\ \frac{m}{2}\end{array}\right)$ para todo $m$ par tal que $2 \leq m \leq n$ si y solo si $T$ es el grafo lineal $L_{n}$.

4. Si $T \neq L_{n}$ entonces $K_{m}(\overleftrightarrow{T})<\left(\begin{array}{c}n-\frac{m}{2} \\ \frac{m}{2}\end{array}\right)$ para algún $m \geq 4$

Además, han planteado como conjetura que, dado un MSD $D$ con $n \geq 2$ vértices y un entero $m$ tal que $2 \leq m \leq n$, entonces la siguiente desigualdad se mantiene:

$$
K_{m}(D) \leq\left(\begin{array}{c}
n-\left\lceil\frac{m}{2}\right\rceil \\
\left\lfloor\frac{m}{2}\right\rfloor
\end{array}\right)
$$

Otro resultado importante es el siguiente.

Lema 7. Si un MSD contiene un ciclo $C_{2}$, entonces los vértices del ciclo son lineales o son puntos de corte.

A continuación, un resultado presentado por los autores sobre la representación de los MSD.

Lema 8. Un MSD se factoriza en un árbol generador con raíz y en un bosque de árboles con raíz invertidos.

Finalmente, afirman que un MSD tiene un árbol dirigido doble subyacente cuyos vértices están dados por la contracción de diagramas de Hasse conexos.

\section{Problemas abiertos}


Otras investigaciones que inciden en nuestro trabajo son las relacionadas al problema espectral. Hay muchos resultados concernientes al problema espectral inverso de matrices no negativas (NIEP), ver $[6,7]$.

Los coeficientes del polinomio característico tienen que ver con cubrimientos por ciclos vía el teorema de los coeficientes. En este sentido, un problema en el que Torres-Mayo et al. se han interesado en [39] es el siguiente: dados los números reales $k_{1}, k_{2}, \ldots, k_{n}$, encontrar condiciones necesarias y suficientes para la existencia de una matriz no negativa $A$ de orden $n$ con polinomio característico $x^{n}+k_{1} x^{n-1}+k_{2} x^{n-2}+\cdots+k_{n}$

Otros problemas en los que se han interesado varios autores son los siguientes:

En 1963, Gallai [35] planteó la siguiente conjetura: Sea $D$ un digrafo fuertemente conexo. Entiéndase el término conjunto estable de $D$ como un subconjunto de vértices que son dos a dos no adyacentes. Sea $\alpha$ la estabilidad de $D$, es decir, el número de vértices máximo de un conjunto estable de $D$. Entonces, todo digrafo fuertemente conexo es generado por $\alpha$ ciclos dirigidos. Esta conjetura fue probada por Thomaseé [36] en el año 2007; la demostración también aplica para los MSD y árboles si se considera, en el último caso, que las aristas son equivalentes a arcos dobles. Existen ejemplos de MSD y árboles donde $\alpha$ ciclos son necesarios para cubrir el correspondiente digrafo: MSD y árboles estrella.

En 1976, Las Vergnas [37] planteó otra conjetura: Sea $D$ un digrafo y sea $\alpha$ el número estabilizador o número de independencia de $D$, es decir, la cardinalidad de un conjunto estable de $D$. Si $\alpha>1$ y $D$ es fuertemente conexo, entonces $D$ es generado por una arborescencia con $\alpha-1$ hojas. Entiéndase por arborescencia un digrafo conexo en el que cada vértice tiene grado de entrada 1, a excepción de la raíz. Esta conjetura también fue probada por Thomaseé [38] en el año 2001. Dicha prueba aplica para los MSD y los árboles si consideramos, en el último caso, que cada arista equivale a arcos dobles. Existen ejemplos de MSD y árboles donde $\alpha-1$ caminos disjuntos son necesarios para cubrir los correspondientes digrafos: MSD y árboles estrella.

\section{Algoritmos de búsqueda de caminos y complejidad}

Bang-Jensen y Gutin [3] y Ausiello et al. [20] describen algunos conceptos y resultados sobre la complejidad de algoritmos para resolver problemas concernientes a grafos y digrafos. A continuación resumimos algunos de ellos. Un problema $\tau$ es llamado problema de decisión si el conjunto $I_{\tau}$ de todas las instancias de $\tau$ es 
dividido en un conjunto $Y_{\tau}$ de instancias positivas y en un conjunto $N_{\tau}$ de instancias negativas y el problema solicita cualquier instancia $x \in I_{\tau}$ para verificar si $x \in Y_{\tau}$. Un problema de decisión $\tau$ es resuelto por un algoritmo $A$ si el algoritmo termina para cada instancia $x \in I_{\tau}$ y devuelve positivo si y solo si $x \in Y_{\tau}$; se dice también que el conjunto $Y_{\tau}$ es reconocido por $A$. Si para un problema existe un algoritmo que lo resuelva en tiempo polinomial, entonces pertenece a la clase polinomial o simplemente clase $P$. Existen otro tipo de problemas para los que se piensa que no existe un algoritmo que solucione su caso más crítico en tiempo polinomial, pero sin embargo si existe un algoritmo que verifica si un candidato a solución es, en efecto, solución en tiempo polinomial. Esta clase de problemas se denominan problemas de complejidad no determinista polinomial o simplemente $N P$ [30]. Existe también otra clase importante de problemas conocidos como NPduro. Un problema es NP-duro si es polinomialmente equivalente a cada problema NP. Un ejemplo de problema NP-duro es el conocido como travelling salesman problem (TSP) que consiste en, dado un digrafo ponderado, encontrar un ciclo hamiltoniano de peso mínimo. Finalmente, si un problema es NP y NP-duro a la vez, entonces se dice que el problema pertenece a la clase NP-completo. Un ejemplo clásico de problema NP-completo es decidir si un digrafo es hamiltoniano.

\section{Algoritmos de búsqueda de caminos de coste mínimo}

Existen algoritmos muy conocidos para buscar caminos con peso mínimo sobre grafos y digrafos, ponderados o no, que se resuelven en tiempo polinomial. A continuación una breve reseña de algunos de ellos, descritos en [3]. El algoritmo Breath-First Search (BFS) permite encontrar rápidamente las distancias (y por lo tanto los caminos) desde un vértice dado $v$ hacia el resto de vértices en digrafos no ponderados. Si el digrafo se encuentra representado por la matriz de adyacencia, la complejidad del algoritmo es $O(n+m)$, siendo $n$ el orden del digrafo y $m$ su tamaño. Para digrafos acíclicos, [3] presenta un algoritmo que permite encontrar los caminos más cortos desde un vértice dado $v$ hacia el resto de vértices en un tiempo $O(n+m)$. El algoritmo de Dijkstra encuentra las distancias desde un vértice dado $v$ hacia el resto de vértices de un digrafo ponderado en un tiempo de $O(n \log n+m)$. El algoritmo Bellman-Ford-Moore permite encontrar las distancias entre un vértice dado $v$ y el resto de vértices de un digrafo ponderado $D$ que puede contener arcos con pesos negativos, pero no ciclos negativos, en un tiempo $O(n m)$. El algoritmo Floyd-Warshall mejora el tiempo alcanzado por Dijkstra 
encontrando todas las distancias en un tiempo de $O\left(n^{3}\right)$.

\section{Otros algoritmos de búsqueda de caminos y ciclos}

Según [21, 23, 24] fue Monien [25] quien presentó la primera aproximación para la búsqueda de caminos largos de longitud $\Omega(\log n / \log \log n)$ en grafos hamiltonianos mediante un algoritmo en tiempo polinomial $O(k ! n m)$ en donde $k$ representa la longitud de un $v_{i} v_{j}$-camino perteneciente al grafo. Este resultado fue mejorado por Fürer y Raghavachari [26, 27] y Karger et al. [28] para encontrar caminos de longitud $\Omega(\log n)$ en grafos hamiltonianos. Karger et al. [28] también señala que el problema del ciclo hamiltoniano aparenta ser duro inclusive en grafos cúbicos y diseñó un algoritmo para encontrar caminos de longitud $\Omega(\sqrt{n} \log n)$ en grafos hamiltonianos cúbicos aleatorios. Alon et al. [29] presentaron un algoritmo para encontrar caminos de longitud $c \log n$ para cualquier valor de $c$ tan grande como sea posible en un grafo dado. Feder et al. [21] desarrollaron un algoritmo para encontrar ciclos cuya longitud sea al menos $m^{\left(\log _{3} 2\right) / 2} \approx m^{0,315}$ en grafos 3 ciclables con $m$ aristas y cuyos vértices tienen como grado máximo 3. Gabow y Nie [23] muestran que el cálculo de caminos y ciclos largos es más costoso en digrafos en comparación al cálculo sobre grafos no dirigidos. Además diseñaron un algoritmo para encontrar un ciclo dirigido de longitud mayor o igual a $\log n / \log \log n$ en tiempo polinomial siempre y cuando exista alguno. Vishwanathan [24] presenta un algoritmo que encuentra un camino de longitud $\Omega\left(\log ^{2} n / \log \log n\right)$ en grafos hamiltonianos no dirigidos. Por otra parte, Björklund et al. [22] afirman que el cálculo de ciclos dirigidos largos es más costoso que el cálculo de caminos dirigidos largos y presenta una modificación del algoritmo de Vishwanathan para aplicarlo en digrafos hamiltonianos.

Los resultados anteriores sobre búsquedas de caminos o ciclos han sido diseñados y probados sobre digrafos con características restringidas o para búsquedas de caminos o ciclos con longitud acotada. Ninguno de ellos ha demostrado funcionar sobre un digrafo arbitrario y tampoco garantizan calcular el camino o ciclo de longitud maximal. 


\section{Capítulo 3}

\section{Estructura de ciclos y vértices en los MSD}

Por definición, comprendemos que un MSD está conformado por un conjunto de vértices y un conjunto de arcos. Se ha mencionado que cada arco debe estar contenido en al menos un ciclo, por lo tanto, un vértice también formará parte de uno o más ciclos del digrafo. Este capítulo presenta los resultados obtenidos en base al estudio de la estructura de los ciclos y los vértices que componen un MSD.

\subsection{Estructura de ciclos}

La configuración de los ciclos de un MSD determina, entre otras cosas, el polinomio característico de su matriz de adyacencia, que es un invariante de la teoría espectral de digrafos $[6,7]$.

La estructura de un ciclo $C_{q}$ de longitud $q$ en un MSD $D$, es decir, la manera en la que el ciclo está incluido en $D$, está determinada por las SC que aparecen en el digrafo $D^{\prime}$ asociado al par $\left(D, C_{q}\right)$ obtenido después de suprimir los arcos del ciclo en $D$. La contracción de estas SC genera un digrafo acíclico que no tiene arcos transitivos debido a que proviene de un MSD. Por lo tanto, puede verse como el diagrama de Hasse de un orden parcial, en el sentido de que contiene la información necesaria para definir una relación de orden entre sus vértices. A este diagrama de Hasse asociado a $\left(D, C_{q}\right)$ lo llamaremos $H$. Un vértice en $H$ es minimal (resp. maximal) si su grado de entrada (resp. grado de salida) es cero. Por lo tanto, los vértices minimales en $H$ corresponden a SC iniciales en 
$D^{\prime}$ y los vértices maximales en $H$ corresponden a $\mathrm{SC}$ finales en $D^{\prime}$. Decimos que un vértice maximal o minimal de $H$ es no trivial si contiene al menos un arco incidente. Llamamos $S C$ trivial a una $\mathrm{SC}$ que contiene solamente un vértice. Una $S C$ anclada en $C_{q}$ es una SC que contiene al menos un vértice de $C_{q}$. Nótese que cada SC es un subdigrafo inducido por el conjunto de sus vértices.

En esta sección analizamos las propiedades de este diagrama de Hasse y sus vértices (SC del digrafo $D^{\prime}$ ).

\subsubsection{Configuración de las SC ancladas}

Sea $D$ un $\mathrm{MSD}, C_{q}$ un ciclo contenido en $D$ y $D^{\prime}$ el digrafo asociado a $\left(D, C_{q}\right)$. $D^{\prime}$ no es fuertemente conexo y sus $\mathrm{SC}$ pueden contener vértices de $C_{q}$, incluso más de uno. Ya hemos comentado que cualquier SC es un digrafo minimal, pues provienen de un MSD.

En este apartado se muestran algunos cálculos exploratorios obtenidos mediante un algoritmo. Este algoritmo ha sido diseñado para calcular exhaustivamente todas las posibles configuraciones de las $\mathrm{SC}$ del digrafo asociado $D^{\prime}$ para $2 \leq q \leq 19$. Se han calculado solamente las configuraciones no isomorfas de las SC del digrafo asociado $D^{\prime}$.

Con el fin de calcular todas las posibles configuraciones de las SC se han considerado todas las posibles inclusiones de los vértices de $C_{q}$ en $\mathrm{SC}$ y se han descartado las configuraciones en las cuales al menos un arco de $C_{q}$ se ha vuelto transitivo. Finalmente se ha calculado y preservado solamente un representante canónico para cada clase de isomorfía.

Pero, antes de proceder a la descripción de los algoritmos, es necesario presentar algunos resultados sobre la estructura de las SC que demostramos a continuación.

Lema 9. [1] Sea $D$ un $M S D, C_{q}$ un ciclo contenido en $D$ y $D^{\prime}$ el digrafo asociado a $\left(D, C_{q}\right)$. Una $S C$ anclada en $C_{q}$ no puede contener vértices consecutivos de $C_{q}$. Así, el número $\lambda$ de vértices de $C_{q}$ en una $S C$ anclada dada satisface $1 \leq \lambda \leq\left\lfloor\frac{q}{2}\right\rfloor$.

Demostración. Supongamos que una $\mathrm{SC}$ tiene dos vértices consecutivos $u$ y $v$. Entonces, el arco $u v$ sería transitivo, contradiciendo el hecho de que $D$ es un MSD.

Ahora, sea $S$ una SC anclada que contiene un número $\lambda$ de vértices de $C_{q}$. Como no pueden ser consecutivos, se tiene que $\lambda \leq\left\lfloor\frac{q}{2}\right\rfloor$. La otra desigualdad, 
$1 \leq \lambda$, es trivial.

La figura 3.1 ilustra esta demostración. En ella se puede apreciar que el arco $u v$ es transitivo.

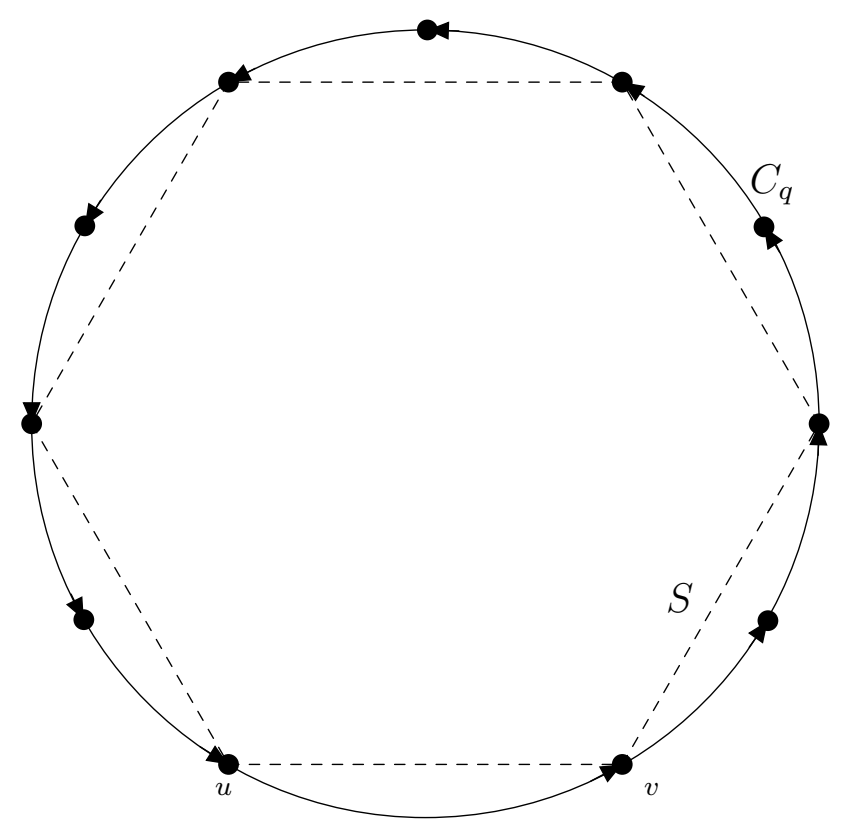

Figura 3.1: Ilustración del Lema 9.

Definición 1. [1] Sea $D$ un $M S D, C_{q}$ un ciclo contenido en $D$ y $D^{\prime}$ el digrafo asociado a $\left(D, C_{q}\right)$. Decimos que dos $S C$ ancladas se cortan si cada una de ellas tiene dos vértices en $C_{q}, u_{1}$ y $u_{2}$ en la primera y $v_{1}$ y $v_{2}$ en la segunda, tal que el orden de esos vértices en el ciclo $C_{q}$ es $u_{1}, \ldots v_{1}, \ldots u_{2}, \ldots v_{2}, \ldots$.

Lema 10. [1] Sea $D$ un $M S D, C_{q}$ un ciclo contenido en $D$ y $D^{\prime}$ el digrafo asociado a $\left(D, C_{q}\right)$. Si dos $S C$ ancladas de $D^{\prime}$ se cortan, estas no pueden tener vértices consecutivos en el ciclo $C_{q}$.

Demostración. Sean $u$ y $v$ vértices consecutivos de $C_{q}$ contenidos en distintas SC de $D^{\prime}, u \in S C_{1}$ y $v \in S C_{2}$, que se cortan. Entonces, el arco $u v$ sería transitivo, contradiciendo el hecho de que $D$ es un MSD.

La figura 3.2 ilustra esta demostración en la que se puede apreciar que el arco $u_{1} v_{1}$ es transitivo. 


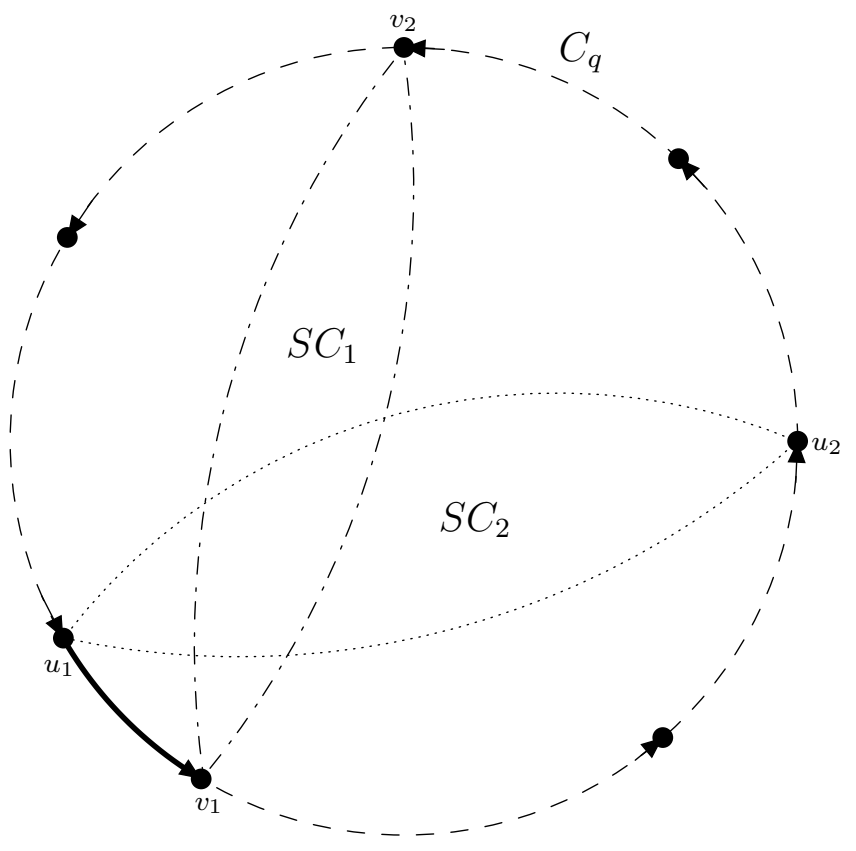

Figura 3.2: Ilustración del Lema 10.

\subsubsection{Consecuencia: Acotación del número de vértices li- neales}

El algoritmo que se ha implementado se presenta a continuación. Cada configuración de SC se representa por un arreglo de números enteros de longitud $q$, que se ha denominado como $C o m p V$ y debe verificar las siguientes propiedades:

1) $C o m p V[k]$ representa la SC a la cual pertenece el vértice $k$.

2) $0 \leq C o m p V[k]<q$ para todo $0 \leq k<q$ (el máximo número de componentes es $q)$.

3) $C o m p V[k] \neq \operatorname{CompV}[(k+1)$ mód $q]$ para todo $0 \leq k<q$ (por el Lema 9$)$.

4) $C o m p V[0]=0$ (dada una configuración de SC, existe siempre una numeración de las SC que verifica esto).

5) $\operatorname{Comp} V[k] \leq 1+\max \{\operatorname{Comp} V[j] \mid 0 \leq j<k\}$ para todo $0<k<q$ (dada una configuración de SC, existe siempre una numeración de las SC que verifica esto). 
Este primer algoritmo implementa la función "Siguiente" que, comenzando en la configuración inicial, genera todas las configuraciones de las SC. Cada vez que es ejecutado calcula la configuración siguiente a la actual. Genera todas las configuraciones de las SC en orden lexicográfico del arreglo Comp V. Las configuraciones iniciales y finales tienen la siguiente forma:

1) Inicial: $[0,1,0, \ldots, 0,1]$ si $q$ es par o $[0,1,0,1, \ldots, 0,1,2]$ si $q$ es impar.

2) Final: $[0,1,2, \ldots, q-2, q-1]$.

Algoritmo 1. Función Siguiente

i) Ubicar (buscando desde $(q-1)$ hasta 0) el primer índice $k$ que permite incrementar el valor de CompV $[k]$. El indice $k$ es el primero que verifica

$$
\operatorname{Comp} V[k] \leq \max \{\operatorname{Comp} V[j] \mid 0 \leq j<k\}
$$

ii) Si $k=0$ devolver NULL (No existen más configuraciones. La configuración actual y la final son las mismas).

iii) Asignar un nuevo valor a CompV $[k]$, $\operatorname{comp}_{k}$ :

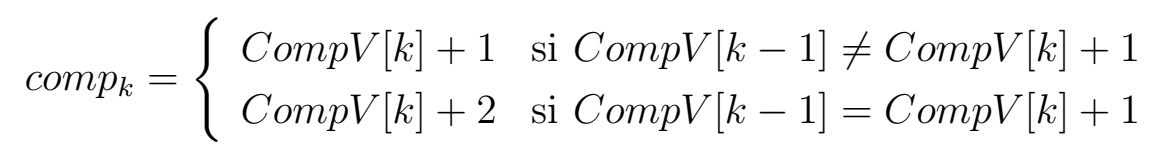

iv) Asignar valores a CompV $[j]$ para $k<j<q$ :

$$
C o m p V[j]=\left\{\begin{array}{l}
0 \text { si } j \neq q-1 \text { y } C o m p V[j-1] \neq 0 \\
1 \quad \text { si } C o m p V[j-1]=0 \\
1 \quad \text { si } j=q-1 \text { y } C o m p V[j-1] \neq 1 \\
2 \quad \text { si } j=q-1 \text { y } C o m p V[j-1]=1
\end{array}\right.
$$

v) Devolver CompV.

El Algoritmo 1 genera todas las configuraciones de SC que verifican el Lema 9, i.e., configuraciones en las que ninguna $\mathrm{SC}$ contiene vértices consecutivos del ciclo $C_{q}$. De acuerdo al Lema 10, las configuraciones que contienen SC que se cortan y tienen vértices consecutivos de $C_{q}$ deben ser descartadas. Es fácil probar 


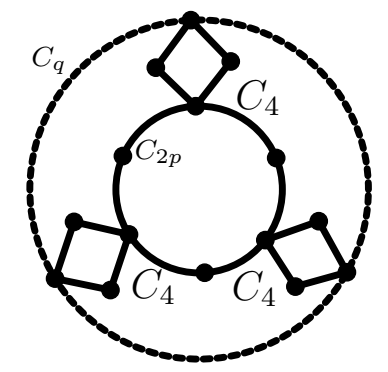

Figura 3.3: Generación de una $\mathrm{SC}$ que contiene $p$ vértices de $C_{q}$.

que cada configuración de SC que verifica los Lemas 9 y 10 representa un MSD. La figura 3.3 muestra una manera de construir las SC.

Para conservar únicamente el representante canónico de cada configuración generada se ha implementado la función "Canónica", que considera los $q$ arreglos que son obtenidos al fijar cada una de las posiciones del arreglo circular CompV como origen. El representante canónico será el más pequeño de ellos, usando el orden lexicográfico.

\section{Algoritmo 2. Función Canónica}

i) Copiar CompV en CompC (CompC será el arreglo canónico).

ii) Para cada $0<k<q$ :

- Calcular Aux $=[C o m p V[k], \ldots, C o m p V[k+q-1]]$ (índices $\bmod q)$.

- Renumerar las SC de Aux de modo que las propiedades de CompV se mantengan:

- Inicializar ReN $[j]=0$ para $0 \leq j<q$ (para indicar si el vértice $j$ está renumerado o no).

- $N e w C o m p=0$.

- Para $j=0$ hasta $j=q-1$ :

$\star$ Si ReN $[j]=0$ (el vértice $j$ no está renumerado), entonces:

* Comp $=$ Aux $[j]$.

* Para $j \leq k<q$ hacer: si Aux $[k]=$ Comp y ReN $[k]=0$ :

$\diamond A u x[k]=N e w C o m p$ y $\operatorname{Re} N[k]=1$.

$* N e w C o m p=N e w C o m p+1$.

- Si Aux $<$ CompC en orden lexicográfico, copiar Aux en CompC. 
iii) Devolver CompC.

La Tabla 3.1 muestra los resultados obtenidos. Notar cómo el número de configuraciones de SC del digrafo asociado $D^{\prime}$ se incrementa exponencialmente a medida que $q$ crece.

\begin{tabular}{rrrr}
\hline$q$ & Núm. de SC & $q$ & Núm. de SC \\
\hline 2 & 1 & 11 & 162 \\
3 & 1 & 12 & 427 \\
4 & 2 & 13 & 1016 \\
5 & 2 & 14 & 2836 \\
6 & 5 & 15 & 7432 \\
7 & 6 & 16 & 20579 \\
8 & 16 & 17 & 52622 \\
9 & 28 & 18 & 159172 \\
10 & 43 & 19 & 449390 \\
\hline
\end{tabular}

Tabla 3.1: Número de configuraciones de SC del digrafo asociado a $\left(D, C_{q}\right)$.

A partir de datos experimentales, se ha llegado a conjeturar que el número de SC ancladas de $D^{\prime}$ tiene $\lfloor(q+3) / 2\rfloor$ como límite inferior.

Sea $S$ una SC del digrafo $D^{\prime}$, denotaremos por $s$ al vértice de $H$ correspondiente. A continuación, se enuncian y demuestran las principales propiedades.

Nota 1. [1] Sea $D$ un $M S D, C_{q}$ un ciclo contenido en $D$ y $D^{\prime}$ y $H$ el digrafo y el diagrama de Hasse asociados a $\left(D, C_{q}\right)$. Sea $s_{1}, \ldots, s_{j}$ un camino en $H$ y u y $v$ vértices de $D$ tal que $u \in S_{1} y v \in S_{j}$. Entonces es obvio que existe un camino de $u$ a $v$ en $D$ que pasa por $S_{1}, \ldots, S_{j-1}$ y $S_{j}$.

Lema 11. Sea $D$ un $M S D, C_{q}$ un ciclo contenido en $D$ y $D^{\prime}$ y $H$ el digrafo y el diagrama de Hasse asociados a $\left(D, C_{q}\right)$. Entonces un ciclo sin vértices lineales en $D$ no puede ser una $S C$ de $D^{\prime}$.

Demostración. En primer lugar, demostramos que una SC $S$ que corresponde a un vértice maximal o minimal de $H$ contiene al menos un vértice de $C_{q}$.

De hecho, si $S$ es un vértice maximal (resp. minimal) en $H$, no tiene arcos de salida (resp. arcos de entrada) desde ningún vértice de $S$ hacia ningún vértice de cualquier otra SC (resp. desde ningún vértice de cualquier otra SC hacia ningún vértice de $S$ ). Existe al menos una $\mathrm{SC}$ diferente de $S$, debido a que $D^{\prime}$ no es 
fuertemente conexo. La conexión fuerte de $D$ implica que existe un arco saliente (resp. entrante) de $S$. Este arco debe pertenecer a $C_{q}$ y consecuentemente $S$ contiene al menos un vértice de $C_{q}$.

Ahora, sea $S$ una SC y sean $u, v \in S$ dos vértices distintos tales que existen los arcos $u a$ y $b v$ en $D$ con $a, b \notin S$. Entonces existe en $D$ un camino de $u$ a $v$ que no contiene arcos de $S$.

En efecto, empecemos un recorrido iniciando en $s_{1}=s$ y caminemos a través de $H$, alejándonos de $s_{1}$ mediante el arco $s_{1} s_{2}$ (en donde $S_{2}$ es la $\mathrm{SC}$ que contiene el vértice $a$ ), hasta que encontremos un vértice maximal de $H: s_{1}, s_{2}, \ldots, s_{j}$. De acuerdo al párrafo anterior, en $S_{j}$ debe existir al menos un vértice de $C_{q}$, llamémoslo $w$. Entonces, según la Nota 1, existe un camino de $u$ a $w$ en $D$. Ahora empezamos el recorrido nuevamente en $s_{1}^{\prime}=s$ y caminamos a través de $H$ en dirección opuesta, alejándonos de $s$ mediante el arco $s_{2}^{\prime} s_{1}^{\prime}$ (en donde $S_{2}^{\prime}$ es la SC que contiene $b$ ), hasta que encontremos un vértice minimal de $H: s_{k}^{\prime}, s_{k-1}^{\prime}, \ldots, s_{2}^{\prime}, s_{1}^{\prime}$. Nuevamente, $S_{k}^{\prime}$ debe contener al menos un vértice de $C_{q}$, sea este $t$. Entonces obtenemos un camino de $t$ a $v$ en $D$ (aplicando la Nota 1 al camino $s_{k}^{\prime}, \ldots, s_{2}^{\prime}, s_{1}^{\prime}$ ). Es obvio que existe un camino de $w$ a $t$ en $C_{q}$. Por tanto, existe un camino de $u$ a $v$ en $D$ (obtenido mediante la concatenación del $u w$-camino con $w t$-camino y $t v$-camino) que no usa arcos de $S$.

Probamos ahora que un ciclo $C$ sin vértices lineales en $D$ no puede ser una $\mathrm{SC}$ de $D^{\prime}$. Sea un ciclo $C$ una $\mathrm{SC}$ de $D^{\prime}$ y consideramos por contradicción que no contiene vértices lineales en $D$. En ese caso, cada vértice de $C$ debe tener un arco de entrada desde o un arco de salida hacia una SC diferente de $C$ o bien estar contenido en $C_{q}$. Así $C$ debe contener al menos dos vértices consecutivos $u$ y $v$ $(u v \in C)$ tales que: 1) $u$ pertenece a $C_{q}$ o existe un arco $u a$ en $D$ con $a \notin C ;$ y 2$)$ $v$ pertenece a $C_{q}$ o existe un arco $b v$ en $D$ con $b \notin C$.

Por lo tanto, es claro, como se ha demostrado anteriormente, que en $D$ existe un camino de $u$ a $v$ que no contiene arcos de $C$. Como consecuencia, el arco $u v$ contenido en $C$ sería transitivo en $D$, en contradicción con la minimalidad de $D$.

La Figura 3.4 ilustra esta demostración, en ella se puede apreciar que el arco $u v$ es transitivo.

Teorema 1. Sea $D$ un $M S D, C_{q}$ un ciclo contenido en $D$ y $D^{\prime}$ y $H$ el digrafo y el diagrama de Hasse asociados a $\left(D, C_{q}\right)$. Entonces, cualquier $S C$ no trivial de $D^{\prime}$ con a lo sumo un vértice de $C_{q}$ tiene al menos un vértice lineal en $D$. 


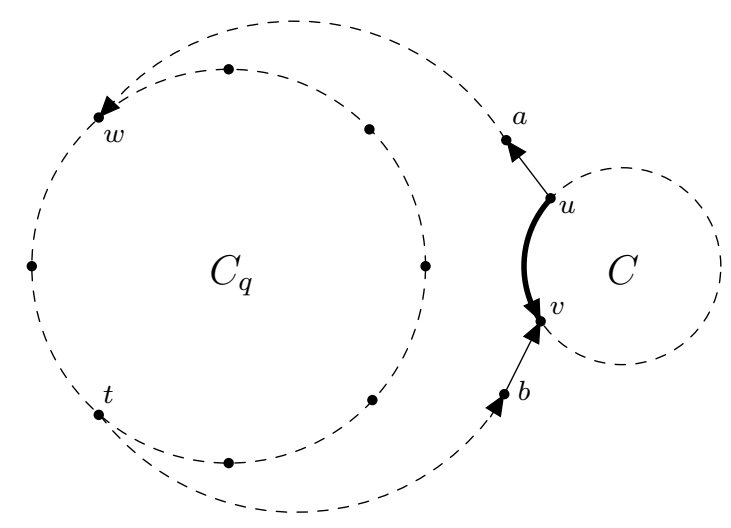

Figura 3.4: Ilustración del Lema 11.

Demostración. Sea $S$ una SC no trivial con a lo sumo un vértice de $C_{q}$. Sea $k$ el número de vértices de $S$. Se demuestra el resultado por inducción sobre $k$.

Paso base: Si $k=2$ entonces $S$ es el ciclo $C_{2}$ y el Lema 11 implica el resultado.

Paso de inducción: Sea $S$ una SC conformada por $k+1$ vértices $(k \geq 2)$ que tiene a lo sumo un vértice de $C_{q}$ y suponemos por hipótesis de inducción que cualquier $\mathrm{SC}$ con a lo sumo $k$ vértices y con a lo sumo un vértice de $C_{q}$ contiene al menos un vértice lineal en $D$.

Si $S$ es el ciclo $C_{k+1}$, el Lema 11 implica el resultado.

Suponemos que $S \neq C_{k+1}$. Entonces $S$ contiene un ciclo $C$ de longitud $p$, $2 \leq p \leq k$. Asumimos que $C$ no contiene ningún vértice lineal en $D$ (en caso contrario, no habría nada que demostrar). $S$ debe contener al menos un vértice que no está contenido en $C$. La contracción de $C$ en un único vértice que llamamos $z$, produce otro MSD $\bar{D}$ que mantiene el ciclo $C_{q}$ (debido a que $S$ contiene a lo sumo un vértice de este ciclo) y otra SC $\bar{S}$ perteneciente al digrafo $\bar{D}^{\prime}$ asociado a $\left(\bar{D}, C_{q}\right)$. El número de vértices de $\bar{S}$ es igual a $k-p+2$, donde $2 \leq k-p+2 \leq k$. Así, por hipótesis de inducción, $\bar{S}$ debe contener al menos un vértice lineal en $\bar{D}^{\prime}$, que llamamos $u$. Ya que $z$ representa a $C$ y éste no tiene vértices lineales, entonces $z \neq u$ porque, en $\bar{D}, d(z) \geq 3$ (si $p=2$, cada vértice de $C_{2}$ tiene un grado mayor o igual a 4 , ya que, por el Lema 7 , son puntos de corte). Por lo tanto, $u$ es un vértice lineal en $D$.

La Figura 3.5 ilustra la demostración.

Nota 2. Sea $D$ un $M S D, C_{q}$ un ciclo contenido en $D$ y $D^{\prime}$ y $H$ el digrafo y el diagrama de Hasse asociados a $\left(D, C_{q}\right)$. Sea uv un arco de H. Entonces las co- 


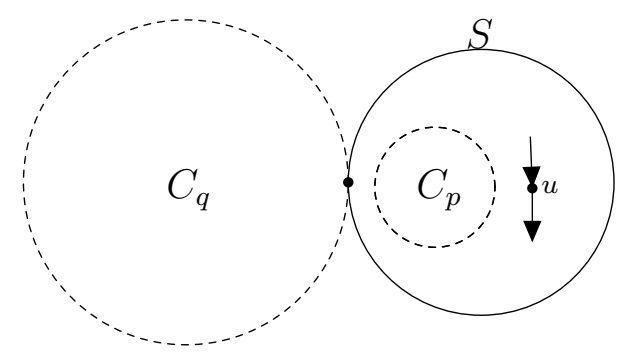

Figura 3.5: Ilustración del Teorema 1.

rrespondientes $S C$ de los vértices $u, v$ de $H$ no pueden contener simultáneamente vértices de $C_{q}$.

Lema 12. Sea $D$ un $M S D, C_{q}$ un ciclo contenido en $D$ y $D^{\prime}$ y $H$ el digrafo $y$ el diagrama de Hasse asociados a $\left(D, C_{q}\right)$. Entonces $H$ tiene al menos un vértice lineal por cada camino entre un vértice minimal y un vértice maximal.

Demostración. Sea $u_{0}, \ldots, u_{j}$ un camino en $H$ que une un vértice minimal $u_{0}$ con un vértice maximal $u_{j}$.

Empezamos recalcando que $j \geq 2$. De hecho, si suponemos que existe un arco en $H$ entre un vértice minimal $u_{0} \mathrm{y}$ un vértice maximal $u_{1}$, en la demostración del Lema 11 hemos probado que ambas SC $U_{0}$ y $U_{1}$ deben contener vértices de $C_{q}$, lo cual es imposible según la Nota 2.

Suponemos ahora, por contradicción, que no existen vértices lineales en el $u_{0} u_{j}$-camino. Así, $d^{-}\left(u_{1}\right)=1$ o caso contrario el arco $u_{0} u_{1}$ sería transitivo en $D$. De hecho, si $v_{1}$ es el vértice minimal alcanzado al caminar en dirección opuesta desde $u_{1}$ utilizando un arco $u_{1}^{\prime} u_{1}$ diferente de $u_{0} u_{1}$ (tal arco existe debido a que $d^{-}\left(u_{1}\right)>1$ ) entonces se puede obtener un camino de $u_{0}$ a $u_{1}$ (que no contiene el arco $\left.u_{0} u_{1}\right)$ mediante la concatenación del $u_{0} v_{1}$-camino con el $v_{1} u_{1}$-camino.

Entonces, $d^{+}\left(u_{1}\right)>1$, ya que $d^{-}\left(u_{1}\right)=1$ y se está asumiendo que $u_{1}$ es un vértice no lineal. Sea $u_{1}^{\prime \prime} \neq u_{2}$ el vértice definido por el arco correspondiente $u_{1} u_{1}^{\prime \prime} \in D$.

Ahora, probamos que $d^{-}\left(u_{i}\right)=1$ para todo $2 \leq i<j$. Para mostrar esto, el siguiente razonamiento se aplica iterativamente para cada vértice, comenzando en $u_{2}$. Primeramente, recordamos que $d^{-}\left(u_{i}\right)=1$. Caso contrario, el arco $u_{i-1} u_{i}$ sería transitivo en $D$, debido a que un camino de $u_{i-1}$ a $u_{i}$ existiría sin contener al arco $u_{i-1} u_{i}$. De hecho, sea $v_{i}$ el vértice minimal que es alcanzado caminando 
en dirección opuesta desde $u_{i}$ comenzando por el arco $u_{i}^{\prime} u_{i}$ (tal arco existe ya que $d^{-}\left(u_{i}\right)>1$ ). Sea $w_{i-1}$ el vértice maximal alcanzado caminando desde $u_{i-1}$ iniciando desde el arco $u_{i-1} u_{i-1}^{\prime \prime}$. Entonces un $u_{i-1} u_{i}$-camino se obtiene mediante la concatenación del $u_{i-1} w_{i-1}$-camino con un $w_{i-1} v_{i}$-camino y el $v_{i} u_{i}$-camino.

Además, $d^{+}\left(u_{i}\right)>1$ también se mantiene, debido a que $d^{-}\left(u_{i}\right)=1 \mathrm{y}$, por hipótesis, $u_{i}$ es un vértice no lineal. Sea $u_{i}^{\prime \prime} \neq u_{i+1}$ el vértice definido por el arco $u_{i} u_{i}^{\prime \prime}$ perteneciente a $H$.

Finalmente, comprobamos que el arco $u_{j-1} u_{j}$ es transitivo. En efecto, sea $w_{j-1}$ el vértice maximal alcanzado caminando desde $u_{j-1}$, empezando con el arco $u_{j-1} u_{j-1}^{\prime \prime}$. Entonces, se obtiene un camino de $u_{j-1}$ a $u_{j}$ mediante la concatenación del $u_{j-1} w_{j-1}$-camino con un $w_{j-1} u_{j}$-camino. Por tanto, alcanzamos una contradicción con la minimalidad de $D$.

La Figura 3.6 ilustra esta demostración.

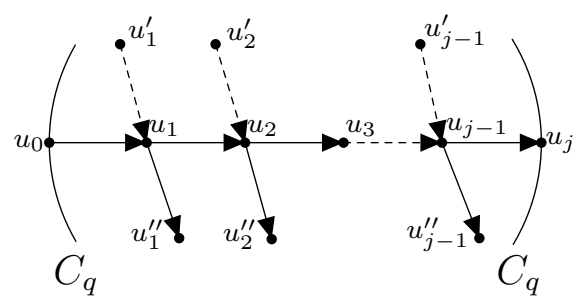

Figura 3.6: Ilustración del Lema 12.

Hay que hacer notar que los vértices lineales resultantes del Lema 12 no pueden ser el primero ni el último del camino original.

Lema 13. Sea $D$ un $M S D, C_{q}$ un ciclo contenido en $D$ y $D^{\prime}$ y $H$ el digrafo $y$ el diagrama de Hasse asociados a $\left(D, C_{q}\right)$. Entonces, la $S C$ correspondiente a un vértice lineal de $H$ contiene al menos un vértice lineal en $D$.

Demostración. Cada SC correspondiente a un vértice de $H$ es un MSD y por tanto contiene al menos dos vértices lineales. De hecho, si la componente no es un MSD lineal, entonces contiene al menos tres vértices lineales. Puesto que, como vértice de $H$ la componente es lineal, solo tiene un arco de entrada y uno de salida. Por tanto, existe al menos un vértice lineal en $D$.

Resta analizar el caso en el que la SC es un MSD lineal y los arcos de entrada y salida inciden en sus dos vértices lineales (si los dos arcos inciden en el mismo vértice, entonces el segundo vértice lineal de la $\mathrm{SC}$ es también lineal en $D$ ). 
Afirmamos que este caso no se puede producir. En efecto, un vértice lineal $u$ de un MSD lineal debe ser parte de un $C_{2}=u, v, u$ o de un $C_{3}=u, v, w, u$. En el primer caso, supongamos que el arco de entrada incide en el vértice $u$. Entonces, podemos construir en $D$ un camino desde $v$ hasta $u$ sin utilizar el arco $v u$ de la siguiente manera: dentro de la componente existe un camino entre $v$ y el vértice de la SC que tiene el arco de salida en $H$ (y que hemos supuesto que es distinto de $u$ ). Puesto que hay un camino en $H$ que empieza con ese arco de salida y llega hasta un maximal, podemos prolongar nuestro camino en $D$ hasta alcanzar un vértice de $C_{q}$. Por otra parte, el vértice $u$ también se puede alcanzar desde $C_{q}$, ya que existe un camino en $H$ que conecta un minimal con la SC que contiene a $u$ y cuyo último arco finaliza en $u$. En conclusión, el arco $v u$ sería transitivo, lo cual es una contradicción.

En el caso de que $u$ esté contenido en un $C_{3}$, un razonamiento completamente análogo nos llevaría a la transitividad del arco wu.

Sea $D$ un MSD, $C_{q}$ un ciclo contenido en $D$ y $D^{\prime}$ y $H$ el digrafo y el diagrama de Hasse asociados a $\left(D, C_{q}\right)$. Llamamos vértice pseudominimal (resp. pseudomaximal) de $H$ a cualquier vértice correspondiente a una $\mathrm{SC}$ anclada con grado de salida (resp. grado de entrada) mayor que 0. Notar que un vértice pseudominimal que no es minimal debe ser también pseudomaximal. Notar también que una SC anclada debe ser pseudominimal, pseudomaximal o trivial. Finalmente, recalcamos que la demostración del Lema 12 es también válida para caminos que empiezan en un vértice pseudominimal y/o terminan en uno pseudomaximal.

Teorema 2. Sea $D$ un $M S D, C_{q}$ un ciclo contenido en $D$ y $D^{\prime}$ y $H$ el digrafo y el diagrama de Hasse asociados a $\left(D, C_{q}\right)$. Entonces, el número de vértices lineales de $H$ es mayor o igual que el número de vértices pseudomaximales (resp. pseudominimales) de $H$.

Demostración. Sea $u_{1}, \ldots, u_{k}$ los vértices pseudominimales de $H$. Para todo $u_{i}$, $1 \leq i \leq k$, considerar un $u_{i} v_{i}$-camino desde $u_{i}$ hasta un pseudomaximal $v_{i}$ de $H$. Aplicando el Lema 12, sea $w_{i}$ el primer vértice lineal que aparece en este camino. Mediante la construcción utilizada en la demostración del Lema 12, se puede demostrar que los vértices $w_{1}, \ldots, w_{k}$ son diferentes dos a dos. De hecho, dado un vértice $u_{i}, 1 \leq i \leq k$, los vértices del $u_{i} w_{i}$-camino no pueden pertenecer a ninguno de los $u_{j} v_{j}$-caminos $(1 \leq j \leq k, j \neq i)$ debido a que el grado de entrada de cada vértice es 1 o $0\left(u_{i}\right)$. Este hecho implica el resultado en el caso 
del pseudominimal. Demostraciones análogas del Lema 12 y del resultado previo, pero iniciando en los vértices pseudomaximales, concluyen la demostración.

Nota 3. El Lema 12 también se cumple si consideramos caminos contenidos en $S C$ en las que todos los ciclos incluyen vértices de $C_{q} y$ con vértices finales en $C_{q}$.

Teorema 3. Sea $D$ un $M S D, C_{q}$ un ciclo contenido en $D$ y $D^{\prime}$ el digrafo asociado $a\left(D, C_{q}\right)$. Una $S C S$ de $D^{\prime}$ que contiene un número $\lambda>1$ de vértices de $C_{q}$ tiene al menos $\lambda$ vértices lineales en $D$.

Demostración. Sean $u_{0}, \ldots, u_{\lambda-1}$ los $\lambda$ vértices de $C_{q}$ pertenecientes a $S$. Sea $\bar{D}$ el MSD obtenido a partir de $D$ después de la contracción iterativa de los ciclos que no incluyen vértices de $C_{q}$. Para cada vértice $u_{i}, 0 \leq i<\lambda$, considerar un $u_{i} u_{i+1}$-camino $\left(i\right.$ módulo $\lambda$ ) en $\bar{D}$. La Nota 3 prueba que cada $u_{i} u_{i+1}$-camino tiene al menos un vértice lineal en $\bar{D}$ y que, si $w_{i}$ es el primer vértice lineal en el $u_{i} u_{i+1}$-camino, el grado de entrada de todos los vértices del $u_{i} w_{i}$-camino (no considerando $u_{i}$ ) es igual a 1 . Esto implica, a su vez, que los vértices $w_{0}, \ldots, w_{\lambda-1}$ son todos diferentes.

Para recuperar la configuración de $D$ podemos expandir iterativamente, en orden inverso, los vértices obtenidos como resultado de la contracción de los ciclos. Durante este proceso podemos actualizar los vértices lineales $w_{i}, 0 \leq i<\lambda$, de la siguiente manera. Sea $x$ el vértice a expandir y $C_{x}$ el ciclo generado como resultado de la expansión. Si $x$ no es un vértice lineal, no hay nada que actualizar. Sin embargo, si $x$ es un vértice lineal, es necesario probar que un nuevo vértice lineal existe en $C_{x}$. Sean $x_{1}$ y $x_{2}$ vértices de $C_{x}$ que definen el arco de entrada y el arco de salida respectivamente en $C_{x}$ (existen exactamente 2 arcos debido a que $x$ es un vértice lineal). Si la longitud de $C_{x}$ es mayor o igual a 3 o la longitud es 2 y $x_{1}=x_{2}$, entonces $C_{x}$ tiene un vértice lineal. Resta analizar el caso en donde $C_{x}$ tiene longitud 2 y $x_{1} \neq x_{2}$. En este caso, tenemos que $C_{x}=C_{2}$, ninguno de los dos vértices de $C_{x}$ es lineal, debido a que ambos tienen un grado total mayor o igual a 3 y ninguno de ellos es un punto de corte debido a que $x_{1}$ y $x_{2}$ están conectados mediante un camino, viniendo desde algún ciclo que pasó a través de $x$ antes de la expansión de $C_{x}$. Esta situación contradice el Lema 7, con lo que se concluye.

La Figura 3.7 ilustra la demostración.

Teorema 4. Sea $D$ un $M S D$ y $C_{q}$ un ciclo contenido en D. Entonces, el número de vértices lineales de $D$ es mayor o igual que $\left\lfloor\frac{q+1}{2}\right\rfloor$. 


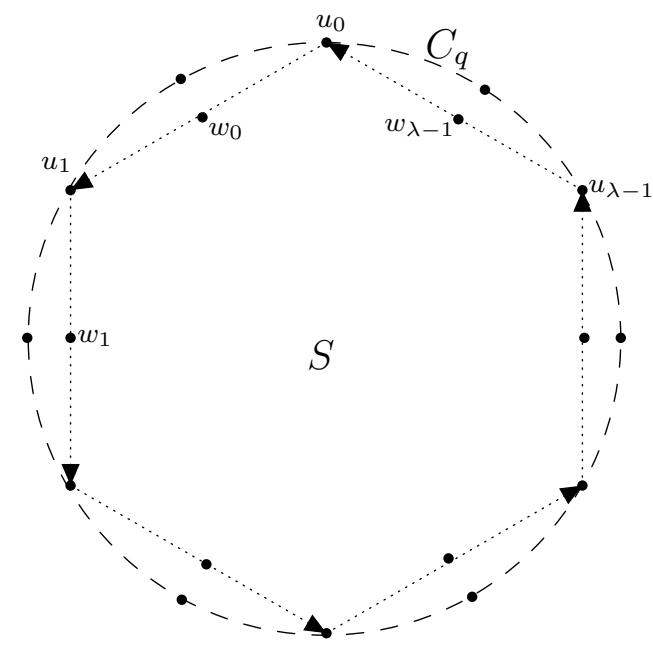

Figura 3.7: Ilustración del Teorema 3.

Demostración. Sea $D^{\prime}$ y $H$ el digrafo y el diagrama de Hasse asociados a $\left(D, C_{q}\right)$. Sea $n_{1}$ el número de vértices de $C_{q}$ que constituyen $\mathrm{SC}$ trivales y que son maximales y minimales a la vez. Sea $\alpha$ el número de vértices lineales de $D$. Cada uno de los vértices de $C_{q}$ no contados en $n_{1}$ pertenece a una $\mathrm{SC}$ no trivial y, por los Teorema 1 y 3, cualquiera contribuye con un vértice lineal. Además, el máximo entre el número de pseudomaximales y el número de pseudominimales de $D^{\prime}$ es mayor o igual que $\left\lceil\frac{n_{1}}{2}\right\rceil$. Por lo tanto, aplicando el Teorema 2 y el Lema 13 , obtenemos

$$
\alpha \geq q-n_{1}+\left\lceil\frac{n_{1}}{2}\right\rceil=q-\left\lfloor\frac{n_{1}}{2}\right\rfloor
$$

El mínimo del término de la derecha de la desigualdad previa es alcanzada cuando $n_{1}=q$ y así

$$
\alpha \geq q-\left\lfloor\frac{q}{2}\right\rfloor=\left\lceil\frac{q}{2}\right\rceil=\left\lfloor\frac{q+1}{2}\right\rfloor
$$

\subsection{Estructura de vértices}

Dado que el grado de entrada y de salida de todo vértice contenido en un MSD es mayor o igual a uno, resulta de interés analizar la estructura en la que los vértices están contenidos en el digrafo; puesto que cada arco que entre o salga de un vértice, por definición de MSD, está contenido en al menos un ciclo. Se han obtenido algunas propiedades de los vértices y su relación con los caminos 
y ciclos de los que forman parte. En esta sección mostraremos estos resultados que hemos conseguido demostrar a través del análisis del grado de los vértices, especialmente aquellos cuyo grado es alto.

\subsubsection{Acotación del número de vértices lineales}

Es claro que la existencia de vértices lineales es un asunto de interés para los MSD. Por ejemplo, resulta trivial demostrar que, a partir de un digrafo fuertemente conexo $D^{\prime}$ en el que existe solamente un arco transitivo, se puede obtener un digrafo $D$ mediante una ampliación interna en ese arco. El vértice añadido es lineal, y el digrafo $D$ es un MSD. El algoritmo para construir un MSD de esta manera se explica más en detalle en la Sección 3.3.3. Es decir, mediante la inserción de vértices lineales dentro de un digrafo fuertemente conexo es posible eliminar los arcos transitivos para obtener un digrafo minimal. Por lo tanto, es interesante estudiar los MSD de tal manera que se puedan determinar los lugares en los que los vértices lineales se encuentran ubicados. En la Sección 3.1.2 hemos realizado este análisis en relación a las SC y en esta sección se presenta el estudio con respecto a los arcos que entran o salen de un vértice.

Un vértice con un grado de entrada (resp. salida) alto estará contenido en una mayor cantidad de ciclos, como en efecto demostraremos más adelante. Como caso extremo, resulta interesante pensar en un vértice que se encuentre contenido en todos los ciclos de un MSD y por lo tanto le corresponda el valor máximo de grado de entrada (resp. salida). Tal caso es posible, por ejemplo, en un MSD con configuración de estrella [7]. Como veremos posteriormente, además, el análisis de este caso nos permitirá obtener conclusiones también para el caso general.

Proposición 1. Sea $D=(V, A)$ un $M S D, \lambda$ el número de vértices lineales de $D$ y $v \in V$ un vértice tal que $v$ está contenido en cada ciclo de D. Entonces, $\lambda \geq \max \left(d^{-}(v), d^{+}(v)\right)$.

Demostración. Si $D$ es un ciclo, entonces $d^{-}(v)=d^{+}(v)=1$. Por lo tanto $\lambda \geq$ $2>\max \left(d^{-}(v), d^{+}(v)\right)=1$ y la demostración concluye.

Sea $C_{q}=v, u_{1}, \ldots, u_{q-1}, v$ un ciclo contenido en $D$. Por definición de MSD, cada arco de $D$ está contenido en al menos un ciclo dirigido de $D$, ya que en caso contrario $D$ no sería fuertemente conexo. Puesto que $v$ está contenido en cada ciclo de $D$, se tiene que cada arco $w u_{i}$ tal que $w \notin C_{q}$ está contenido en un ciclo 
$v, \ldots, w, u_{i}, \ldots, v$ para $1 \leq i \leq q-1$. De manera similar, cada arco $u_{i} w$ tal que $w \notin C_{q}$ está contenido en un ciclo $v, \ldots, u_{i}, w, \ldots, v$ para $1 \leq i \leq q-1$.

Ahora probaremos que en $C_{q}$ debe existir al menos un vértice lineal. De hecho supongamos, por contradicción, que $u_{i} \in C_{q}$ no es un vértice lineal para ningún $1 \leq i \leq q-1$. Así, $d^{-}\left(u_{1}\right)=1$, pues en caso contrario el arco $v u_{1}$ sería transitivo en $D$. En efecto, si $d^{-}\left(u_{1}\right)>1$, puesto que $v$ está contenido en cada ciclo, el vértice $v$ se puede alcanzar avanzando en sentido contrario desde $u_{1}$ utilizando el arco $u_{1}^{\prime} u_{1}$ distinto de $v u_{1}$ (tal arco existe ya que $d^{-}\left(u_{1}\right)>1$ ). En consecuencia, podríamos construir un $v u_{1}$-camino (que no contiene el arco $v u_{1}$ ) mediante la concatenación del $v u_{1}^{\prime}$-camino con el arco $u_{1}^{\prime} u_{1}$.

Entonces debe ser $d^{+}\left(u_{1}\right)>1$, puesto que $d^{-}\left(u_{1}\right)=1$ y estamos asumiendo que $u_{1}$ no es lineal. Sea $u_{1}^{\prime \prime} \neq u_{2}$ un vértice definido por el correspondiente arco $u_{1} u_{1}^{\prime \prime} \in D$.

A continuación, probamos el siguiente resultado para todo $u_{i}, 2 \leq i \leq q-1$ : $d^{-}\left(u_{i}\right)=1$ y existe un arco $u_{i} u_{i}^{\prime \prime}$ con $u_{i}^{\prime \prime} \neq u_{i+1}$. Para probarlo, el siguiente razonamiento se aplica iterativamente para cada vértice, comenzando en $u_{2}$. Primeramente, remarquemos el hecho de que $d^{-}\left(u_{i}\right)=1$. De otra manera, el arco $u_{i-1} u_{i}$ sería transitivo en $D$, porque existiría un $u_{i-1} u_{i}$-camino que no contiene el arco $u_{i-1} u_{i}$. En efecto, puesto que el vértice $v$ está contenido en cada ciclo, el vértice $v$ se puede alcanzar avanzando en sentido opuesto desde $u_{i}$, comenzando con el arco $u_{i}^{\prime} u_{i}$, distinto de $u_{i-1} u_{i}$ (tal arco existe, puesto que $d^{-}\left(u_{i}\right)>1$ ). A su vez, el vértice $v$ también puede ser alcanzado desde $u_{i-1}$, comenzando con el arco $u_{i-1} u_{i-1}^{\prime \prime}$. En consecuencia, se podría obtener un $u_{i-1} u_{i}$-camino mediante la concatenación del arco $u_{i-1} u_{i-1}^{\prime \prime}$ con el $u_{i-1}^{\prime \prime} v$-camino, el $v u_{i}^{\prime}$-camino y el arco $u_{i}^{\prime} u_{i}$.

Por otra parte, $d^{+}\left(u_{i}\right)>1$ también se mantiene, porque $d^{-}\left(u_{i}\right)=1 \mathrm{y}$, por hipótesis, $u_{i}$ no es un vértice lineal. Sea $u_{i}^{\prime \prime} \neq u_{i+1}$ un vértice definido por el arco $u_{i} u_{i}^{\prime \prime}$ perteneciente a $D$.

Finalmente, probamos que el arco $u_{q-1} v$ es transitivo. En efecto, puesto que el vértice $v$ está contenido en cada ciclo, se puede alcanzar avanzando desde $u_{q-1}$, iniciando por el arco $u_{q-1} u_{q-1}^{\prime \prime}$. El $u_{q-1} v$-camino obtenido mediante la concatenación del arco $u_{q-1} u_{q-1}^{\prime \prime}$ con el $u_{q-1}^{\prime \prime} v$-camino prueba que $u_{q-1} v$ es transitivo. Este hecho contradice la minimalidad de $D$.

Para completar la prueba, resta aún demostrar que los vértices lineales alcanzados por cada arco de salida (resp. arco de entrada) desde (resp. hacia) $v$ son todos distintos. Sean $v u_{1}$ y $v u_{1}^{\prime}$ dos arcos en $D$. Desde $v u_{1}^{\prime}$, como hemos visto, po- 


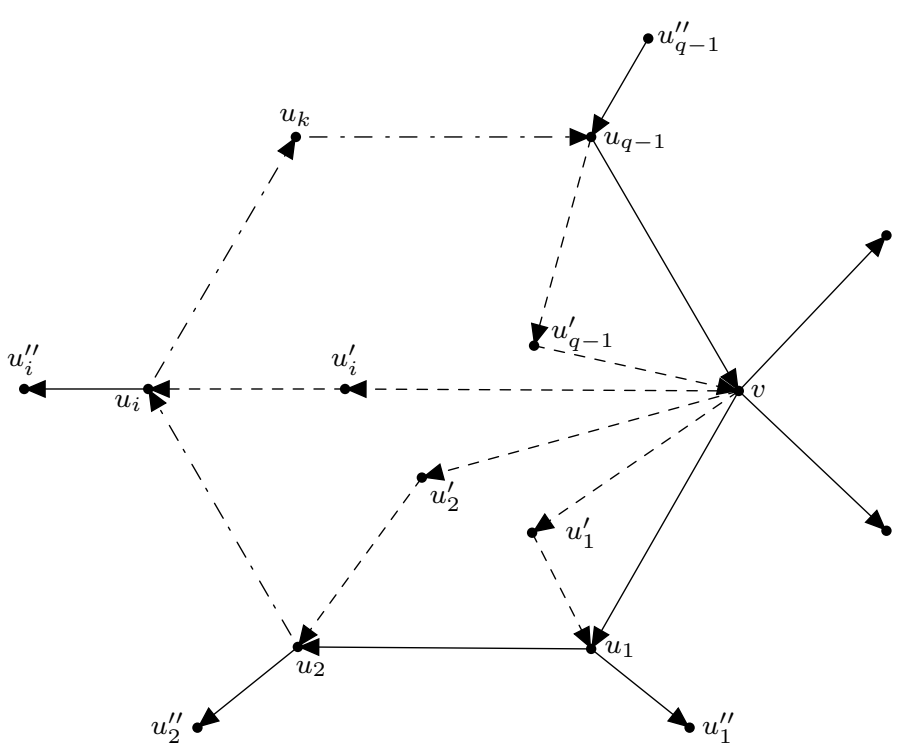

Figura 3.8: Ilustración de la Proposición 1.

demos construir un camino $v, u_{1}, \ldots, u_{k}$ tal que $d^{-}\left(u_{i}\right)=1$ para todo $1 \leq i \leq k$, $d^{+}\left(u_{i}\right)>1$ para todo $1 \leq i \leq k-1$ y $u_{k}$ es lineal (notar que $k$ puede ser 1 , pero en todo caso debe existir). De manera análoga, construimos un camino $v, u_{1}^{\prime}, \ldots, u_{l}^{\prime}$ tal que $d^{-}\left(u_{j}^{\prime}\right)=1$ para todo $1 \leq j \leq l$ y $d^{+}\left(u_{j}^{\prime}\right)>1$ para todo $1 \leq j \leq l-1$ y $u_{l}^{\prime}$ es lineal. Los caminos $v, u_{1}, \ldots, u_{k} \mathrm{y} v, u_{1}^{\prime}, \ldots, u_{l}^{\prime}$ no pueden confluir después de abandonar el vértice $v$, puesto que todos los grados de entrada de sus vértices son 1. Por lo tanto, $u_{k} \neq u_{l}^{\prime}$.

Proposición 2. Sea $D=(V, A)$ un $M S D$ de orden $n \geq 2, v \in V$ un vértice de $D$ y $\lambda$ el número de vértices lineales de $D$. Entonces $\lambda \geq \max \left(d^{-}(v), d^{+}(v)\right)$.

Demostración. Si $D=C_{n}$ se tiene que $d^{-}(v)=d^{+}(v)=1$ para todos los vértices $v$. En consecuencia $\lambda=n \geq 1$ y el resultado se verifica trivialmente.

En caso contrario, si $D$ no es un ciclo, obtenemos un MSD $D^{\prime}$ a partir de $D$ mediante la contracción sucesiva de todos los ciclos que no contienen al vértice $v$. Hacemos notar que $v$ es un vértice contenido en cada ciclo de $D^{\prime}$. Por lo tanto, podemos usar la Proposición 1 para concluir que $\lambda^{\prime} \geq \max \left(d^{-}(v), d^{+}(v)\right)$, donde $\lambda^{\prime}$ es el número de vértices lineales de $D^{\prime}$. Nótese también que en $D^{\prime}$ se preservan todos los arcos de $D$ que inciden en el vértice $v$. Afirmamos entonces que en el proceso de deshacer en orden inverso todas las contracciones de ciclos, para volver a obtener el digrafo $D$ a partir del digrafo $D^{\prime}$, el número de vértices lineales se 
mantiene o crece. En efecto, si expandimos un vértice lineal correspondiente a un ciclo de longitud mayor que 2 , es obvio que en el ciclo expandido hay al menos un vértice lineal (de hecho, si el ciclo es de longitud $q$, tiene $q-2$ vértices lineales). Y si expandimos un vértice lineal correspondiente a un ciclo de longitud 2, el resultado es consecuencia del Lema 7.

En el ejemplo de la figura 3.9 se puede apreciar el proceso de contracción de todos los ciclos (vértices verdes) que no tocan el vértice $v$. Y, en la figura 3.10 se muestra el proceso inverso en el que se encuentra un vértice lineal (azul) por cada arco de salida de $v$.
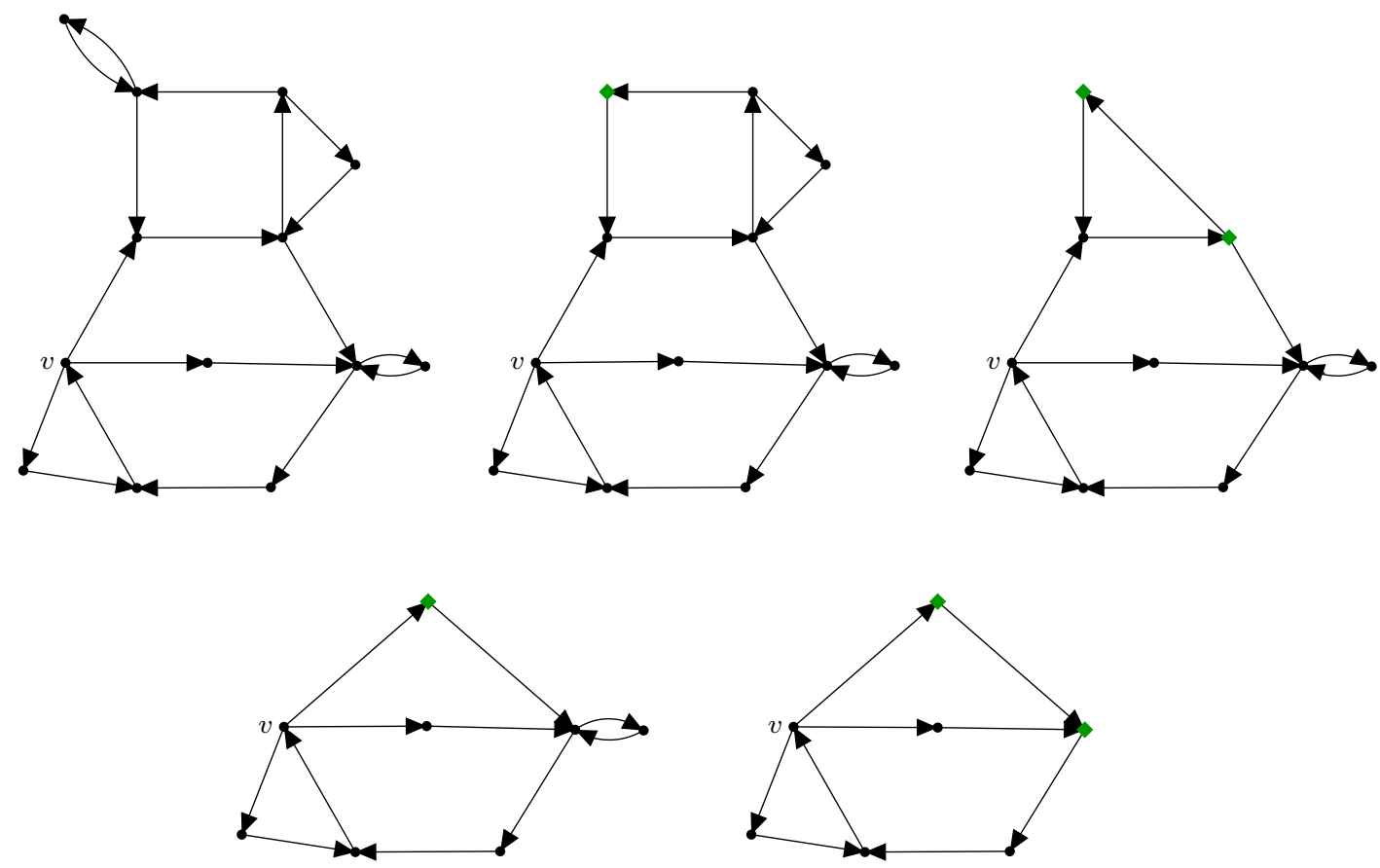

Figura 3.9: Ilustración de la Proposición 2. Ejemplo de construcción de un MSD en el que existe un vértice contenido en todos los ciclos.

Dado un ciclo, podemos definir su grado de entrada (resp. salida) como el grado de entrada del vértice obtenido por la contracción del ciclo. El siguiente resultado es consecuencia directa de la Proposición 2.

Corolario 1. Sea $D=(V, A)$ un $M S D, C_{q}$ un ciclo contenido en $D$ y $\mu$ el número de vértices lineales de $D$ no contenidos en $C_{q}$. Entonces $\mu \geq \max \left(d^{-}\left(C_{q}\right), d^{+}\left(C_{q}\right)\right)$.

Demostración. Si $D=C_{q}$, se tiene que $\mu=d^{-}\left(C_{q}\right)=d^{+}\left(C_{q}\right)=0$ y el resultado se verifica. 

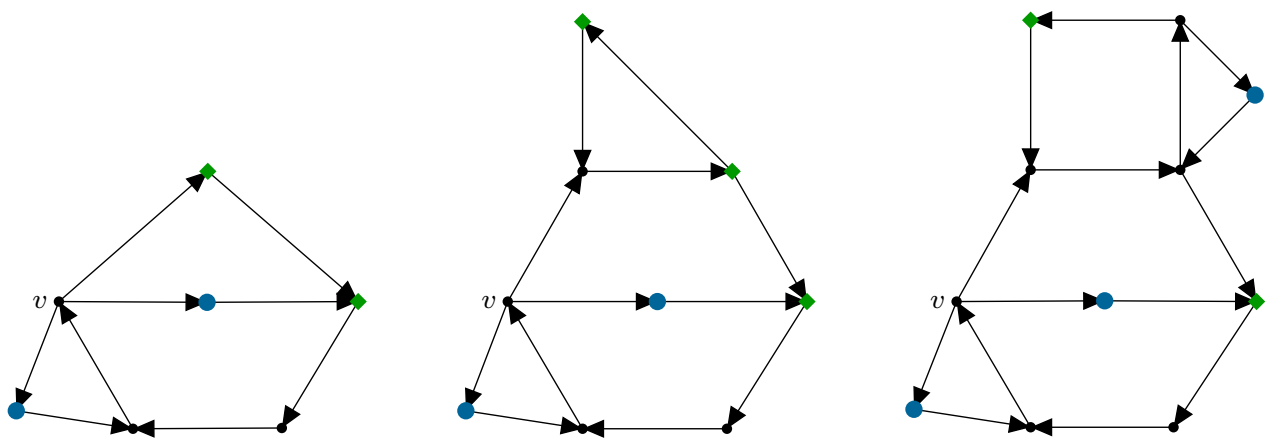

Figura 3.10: Ilustración de la ubicación de los vértices lineales en el MSD de la figura 3.9 .

En caso contrario, obtenemos un MSD $D^{\prime}$ a partir de $D$ mediante la contracción de $C_{q}$ en un único vértice $v^{\prime}$. Utilizando la Proposición 2 podemos afirmar que $\mu^{\prime} \geq \max \left(d^{-}\left(v^{\prime}\right), d^{+}\left(v^{\prime}\right)\right)$, donde $\mu^{\prime}$ es el número de vértices lineales de $D^{\prime}$. Nótese que $d^{-}\left(C_{q}\right)=d^{-}\left(v^{\prime}\right)$ y $d^{+}\left(C_{q}\right)=d^{+}\left(v^{\prime}\right)$. Por lo tanto, $\mu^{\prime} \geq \max \left(d^{-}\left(C_{q}\right), d^{+}\left(C_{q}\right)\right)$. Finalmente, es evidente que cada vértice lineal $u \in D^{\prime}$ distinto de $v^{\prime}$ es un vértice lineal en $D$ que no pertenece a $C_{q}$.

\subsubsection{Consecuencia en la longitud de los ciclos}

Como se ha dicho en el Teorema 4, si existe un ciclo $C_{q} \in D$, el número de vértices lineales de $D$ es mayor o igual que $\left\lfloor\frac{q+1}{2}\right\rfloor$ (puede verse también en [1]). Estamos en disposición de dar una demostración nueva más corta de este resultado, como corolario de las propiedades que acabamos de demostrar.

Corolario 2. Sea $D=(V, A)$ un $M S D$ de orden $n \geq 2, C_{q}$ un ciclo contenido en $D$ y $\lambda$ el número de vértices lineales de $D$. Entonces $\lambda \geq\left\lfloor\frac{q+1}{2}\right\rfloor$.

Demostración. Sea $\nu$ el número de vértices lineales contenidos en $C_{q}$ y $\mu$ el resto de vértices lineales de $D$. Entonces, $\lambda=\mu+\nu$ y sabemos por el Corolario 1 que $\mu \geq \max \left(d^{+}\left(C_{q}\right), d^{-}\left(C_{q}\right)\right)$. Puesto que $d^{+}\left(C_{q}\right)+d^{-}\left(C_{q}\right) \geq q-\nu$, tenemos que

$$
\mu \geq \max \left(d^{+}\left(C_{q}\right), d^{-}\left(C_{q}\right)\right) \geq\left\lceil\frac{q-\nu}{2}\right\rceil
$$

y en consecuencia

$$
\lambda=\mu+\nu \geq\left\lceil\frac{q-\nu}{2}\right\rceil+\nu=\left\lceil\frac{q+\nu}{2}\right\rceil \geq\left\lceil\frac{q}{2}\right\rceil=\left\lfloor\frac{q+1}{2}\right\rfloor .
$$


Como consecuencia del Corolario 2, obtenemos una cota superior para la longitud máxima de un ciclo contenido en un MSD.

Corolario 3. Sea $D=(V, A)$ un $M S D$ de orden $n \geq 2, C_{l}$ un ciclo con longitud maximal $l$ contenido en $D$ y $\lambda$ el número de vértices lineales de D. Entonces $l \leq 2 \lambda$.

Demostración. Por el Corolario 2 sabemos que

$$
\lambda \geq\left\lfloor\frac{l+1}{2}\right\rfloor
$$

y entonces

$$
l \leq 2 \lambda
$$

Proposición 3. Sea $D=(V, A)$ un $M S D$ y sea $C_{q}$ un ciclo de longitud $q$ contenido en $D$. Entonces $q \leq 2 n-m$.

Demostración. Obtenemos un MSD $D^{\prime}$ mediante la contracción de $C_{q}$ en un único vértice $v^{\prime}$. Se tiene entonces que $n^{\prime}=n-q+1$ y $m^{\prime}=m-q$. Por lo tanto, puesto que

$$
m^{\prime} \leq 2\left(n^{\prime}-1\right)
$$

obtenemos que

$$
m-q \leq 2(n-q)
$$

y finalmente

$$
q \leq 2 n-m
$$

\subsection{Otros resultados}

En $[3,7]$ pueden encontrarse algunos resultados relacionados con la descomposición en orejas de un digrafo fuertemente conexo. Hemos utilizado algunos de ellos para demostrar las siguientes propiedades de los MSD. 


\subsubsection{Cadenas lineales externas}

Definición 2. Sea $D=(V, A)$ un $M S D$ de orden $n \geq 2$ y sea $v_{1}, \ldots, v_{l}$ un camino contenido en $D$. Decimos que el $v_{1} v_{l}$-camino es una cadena lineal de longitud $l$ si $d^{-}\left(v_{i}\right)=d^{+}\left(v_{i}\right)=1$ para todo $1 \leq i \leq l$.

Nótese que un vértice lineal aislado es una cadena lineal de longitud 1.

Definición 3. Sea $D=(V, A)$ un $M S D$ de orden $n \geq 2$, sea $v_{1}, \ldots, v_{l}$ una cadena lineal contenida en $D$ y sea $D^{\prime}$ el digrafo obtenido a partir de $D$ mediante la eliminación del $v_{1} v_{l}$-camino. Decimos que el $v_{1} v_{l}$-camino es una cadena lineal externa con longitud $l$ si $D^{\prime}$ es fuertemente conexo.

Lema 14. Sea $D=(V, A)$ un $M S D$ de orden $n \geq 2$ y sea $C_{q}$ un ciclo contenido en $D$ tal que $D \neq C_{q}$. Entonces, en $D$ existe al menos una cadena lineal externa.

Demostración. Usamos de manera análoga la descomposición en orejas descrita en [7, Theorem 20], que prueba que un MSD se factoriza en un árbol con raíz y en un bosque de árboles con raíz invertidos.

Consideremos una descomposición en orejas de $D, \mathcal{E}=P_{0}, \ldots, P_{k}$. Puesto que $D$ es un MSD, cada oreja $P_{j}(0 \leq j \leq k)$ contiene al menos un vértice nuevo y dos arcos nuevos, con respecto a $\bigcup_{i=0}^{j-1} V_{i}$ y $\bigcup_{i=0}^{j-1} A_{i}$, respectivamente.

En consecuencia, es claro que la última oreja $P_{k}=v_{0}^{k} \ldots v_{s_{k}}^{k}$ completa la construcción de $D$ y $Q_{k}=v_{1}^{k} \ldots v_{s_{k}-1}^{k}$ debe ser, por tanto, una cadena de vértices lineales, cuyo primer y último vértice están unidos a vértices de un digrafo $D^{\prime}$ que es minimal y fuertemente conexo. Así, $D^{\prime}=D-Q_{k}$ es un MSD y por lo tanto $Q_{k}$ es una cadena lineal externa de longitud $s_{k}-1 \geq 1$. Trivialmente podemos decir que si $D=C_{q}=P_{0}$, entonces no existen cadenas lineales externas contenidas en $D$.

Nótese que, según el Lema $14, D^{\prime}$ es un MSD con $n-l$ vértices y $m-l-1$ arcos. Notar también que si $P_{0}=C_{q}$, siendo $C_{q}$ un ciclo de longitud maximal contenido en $D$, entonces la cadena lineal externa con longitud $l$ no estaría contenida en $C_{q}$. En ese caso, $q \leq n-l$, en donde $l \geq 1$.

Lema 15. Sea $D=(V, A)$ un $M S D$ y sea el $v_{1} v_{l}$-camino una cadena lineal contenida en $D$ con longitud $l<n$. Entonces la contracción de todos los vértices del $v_{1} v_{l}$-camino en un único vértice preserva la minimalidad, esto es, produce otro $M S D D^{\prime}$ con $n-l+1$ vértices y $m-l+1$ arcos. 
Demostración. Sea $D^{\prime}$ el digrafo obtenido mediante la contracción de todos los vértices del $v_{1} v_{l}$-camino en un único vértice $v^{\prime}$. Sea $n^{\prime}$ el orden y $m^{\prime}$ el tamaño de $D^{\prime}$ respectivamente. En $D^{\prime}$ todos los vértices del $v_{1} v_{l}$-camino están suprimidos pero contiene el vértice $v^{\prime} \notin D$, entonces $n^{\prime}=n-l+1$. Puesto que $d^{-}\left(v_{i}\right)=d^{+}\left(v_{i}\right)=1$ para todo $1 \leq i \leq l$, tenemos que $m^{\prime}=m-l+1$.

Supongamos que existen arcos transitivos en $D^{\prime}$. Si expandimos nuevamente $v^{\prime}$, aquellos arcos transitivos deberían también existir en $D$, contradiciendo la minimalidad de $D$.

Además, puesto que $n>l$, sabemos que debe existir un vértice $w$ de $D$, tal que $w \notin v_{1} v_{l}$-camino. El vértice $w$ también pertenece a $D^{\prime}$ y es evidente que $D^{\prime}$ contiene un $w v^{\prime}$-camino y un $v^{\prime} w$-camino. Por lo tanto $D^{\prime}$ es fuertemente conexo. Como $D^{\prime}$ no tiene arcos transitivos, es minimal.

Lema 16. Sea $D=(V, A)$ un $M S D$ tal que $D$ no es un ciclo. Entonces no existe un ciclo en $D$ que contenga todos los vértices lineales de $D$.

Demostración. Suponemos que $C_{q}$ contiene todos los vértices lineales de $D$. Podemos obtener un MSD $D^{\prime}$ mediante la contracción de $C_{q}$ en un único vértice $v^{\prime}$. Sabemos que $D^{\prime}$ debe contener al menos dos vértices lineales y al menos uno de ellos es distinto de $v^{\prime}$. En consecuencia, es claro que existe al menos un vértice lineal que está contenido en $D$ pero no en $C_{q}$.

Sea $D$ un MSD tal que $D$ no es un ciclo y $\lambda$ el número de vértices lineales de $D$. Nótese que, a partir del Lema 16 , es trivial demostrar que un ciclo $C_{q}$ contenido en $D$ podrá contener como máximo $\lambda-1$ vértices lineales de $D$.

\subsubsection{Coeficientes del polinomio característico}

En [7] se demuestran algunos resultados acerca de cotas para los coeficientes del polinomio característico de un MSD. En particular, se demuestra que el término independiente debe ser 1, 0 o -1. Seguimos las líneas de esa demostración para generalizar esa cota.

Proposición 4. Sea $D=(V, A)$ un $M S D$ y sea $x^{n}+k_{1} x^{n-1}+\cdots+k_{i} x^{n-1}+\cdots+$ $k_{n-1} x+k_{n}$ el polinomio característico de la matriz de adyacencia de D. Entonces

$$
\left|k_{i}\right| \leq\left(\begin{array}{c}
n \\
i
\end{array}\right)
$$


Demostración. Teniendo el cuenta el Teorema de los coeficientes, es suficiente con probar que cualquier subconjunto $A$ de $i$ vértices del MSD $D$ puede ser cubierto mediante ciclos disjuntos a lo sumo de una manera. Sea $D^{\prime}$ el subdigrafo inducido por $A$. Entonces, $D^{\prime}$ es un subdigrafo de un MSD, así que no contiene arcos transitivos. Si $D^{\prime}$ es SC, entonces es un MSD. En caso contrario, podemos añadir arcos hasta obtener un digrafo fuertemente conexo $D^{\prime \prime}$ que sería un MSD. Por el resultado de [7] anteriormente citado, existe a lo sumo un cubrimiento de los vértices de $D^{\prime \prime}$ (o de $D^{\prime}$, caso de ser MSD) mediante ciclos disjuntos. Recordemos que los vértices de $D^{\prime \prime}$ ( y los de $D^{\prime}$ ) son los de $A$. Como cualquier cubrimiento de $A$ en $D$ mediante ciclos disjuntos sería también un cubrimiento de $A$ en $D^{\prime \prime}$ (las aristas del cubrimiento en $D$ pertenecen a $D^{\prime \prime}$ ), se concluye que $A$ no puede tener dos cubrimientos distintos en $D$.

\subsubsection{Algoritmos de búsqueda de ciclos de longitud máxi- ma}

Es bien conocido que la minimalidad es una condición muy estricta en la familia de los digrafos fuertemente conexos. Por ejemplo, implica la limitación del tamaño $n \leq m \leq 2(n-1)$ y, como hemos visto en las secciones previas, los MSD también presentan restricciones fuertes sobre el número de vértices lineales y el máximo grado de entrada (resp. salida) de sus vértices, en relación a la longitud del ciclo dirigido más largo. Desafortunadamente, esas restricciones no son suficientes para construir un algoritmo eficiente que calcule el ciclo más largo en un MSD.

Teorema 5. El cálculo de un ciclo con longitud máxima en un MSD es un problema NP-hard.

Demostración. Podemos reducir el problema del cálculo de un ciclo con longitud máxima en un digrafo fuertemente conexo al problema de calcular el ciclo con longitud máxima en un MSD.

Sea $D^{\prime}=\left(V^{\prime}, A^{\prime}\right)$ un digrafo fuertemente conexo. Podemos construir un MSD $D=(V, A)$ a partir de $D^{\prime}$ de la siguiente manera. Para cada arco $v_{i}^{\prime} v_{j}^{\prime} \in A^{\prime}$ añadimos un vértice intermedio $v_{i j}$. Así obtenemos

$$
V=V^{\prime} \cup\left\{v_{i j} \mid v_{i}^{\prime} v_{j}^{\prime} \in A^{\prime}\right\}
$$




$$
A=\left\{v_{i}^{\prime} v_{i j} \mid v_{i}^{\prime} v_{j}^{\prime} \in A^{\prime}\right\} \cup\left\{v_{i j} v_{j}^{\prime} \mid v_{i}^{\prime} v_{j}^{\prime} \in A^{\prime}\right\} .
$$

Hay que hacer notar que la conexión fuerte de $D^{\prime}$ implica que $D$ es también fuertemente conexo. Notar además que ningún arco de $D$ puede ser transitivo, puesto que cada arco tiene un vértice lineal $v_{i j}$ como extremo inicial o final. Por lo tanto, $D$ es de hecho un MSD.

Ahora, remarcamos que existe una correspondencia uno a uno entre los ciclos de $D$ y los ciclos de $D^{\prime}$ : para cada ciclo $C_{q}^{\prime}$ en $D^{\prime}$, un ciclo $C_{2 q}$ surge en $D$ y todos los ciclos en $D$ son generados de esta manera.

Concluimos que cualquier algoritmo que permita calcular el ciclo más largo de un MSD debería permitir también calcular el ciclo más largo en cualquier digrafo fuertemente conexo. Concluimos observando que el problema de calcular el ciclo más largo en un digrafo fuertemente conexo es NP-hard [3].

Teorema 6. Sea $D=(V, A)$ un $M S D$. Entonces, determinar si $D$ contiene un ciclo de longitud $2 n-m$ es un problema $N P$-completo.

Demostración. Podemos reducir el problema de determinar si un digrafo es hamiltoniano al problema de determinar si un MSD contiene un ciclo de longitud $2 n-m$.

Sea $D^{\prime}=\left(V^{\prime}, A^{\prime}\right)$ un digrafo. Si $D^{\prime}$ es fuertemente conexo, el mismo procedimiento utilizado en el Teorema 5 produce un $\operatorname{MSD} D=(V, A)$ (si $D^{\prime}$ no es fuertemente conexo, entonces no puede ser hamiltoniano). El orden de $D$ verifica $n=n^{\prime}+m^{\prime}$ y su tamaño es $m=2 m^{\prime}$. Por lo tanto, encontrar un ciclo en $D$ con longitud $2 n-m=2\left(n^{\prime}+m^{\prime}\right)-2 m^{\prime}=2 n^{\prime}$ implicaría encontrar un ciclo $C_{n^{\prime}}$ en $D^{\prime}$, esto es, determinar si $D$ es hamiltoniano. Basta entonces la observación de que determinar si un digrafo es hamiltoniano es un problema NP-completo. 


\section{Capítulo 4}

\section{Conclusiones}

A partir de los algoritmos implementados para la obtención de todas las configuraciones posibles de las SC que aparecen al eliminar los arcos de un ciclo contenido en un MSD y de la construcción del diagrama de Hasse asociado, podemos decir que la configuración de un ciclo en un MSD está restringida por varias condiciones que garantizan que el digrafo mantenga las propiedades de conexión fuerte y minimalidad. Resultaría interesante, en un futuro, estudiar con mayor profundidad la estructura de este diagrama de Hasse, con el fin de caracterizarlo, pues también podría presentar varias restricciones.

Un MSD lineal contiene exactamente dos vértices lineales y la longitud del ciclo más corto es menor o igual que 4. Hemos generalizado esta observación, dando una cota inferior para el número de vértices lineales de un MSD a partir de la longitud de cualquier ciclo contenido en él. Queda abierto el problema de estudiar la caracterización de los MSDs para los que la cota es ajustada.

En el caso de los árboles, cada vértice de grado alto implica la aparición de más hojas. Esta observación también ha sido generalizada para los MSD, acotando inferiormente el número de vértices lineales en función del grado de salida (o de entrada) de un vértice contenido en un MSD. Sería de interés estudiar si es posible determinar o acotar inferiormente el número de vértices lineales de un MSD en función de los in- o exgrados de todos sus vértices.

Puesto que el número de vértices lineales contenidos en un MSD puede ser calculado de manera eficiente, podríamos aprovechar el hecho de la acotación de la longitud del ciclo más largo en función del número de vértices lineales (recíproco), para acotar la búsqueda de un ciclo de longitud maximal contenido en un MSD; pero, hemos demostrado que este problema es NP-duro, por lo tanto, esto es 
imposible. Sin embargo, en un futuro, se podría caracterizar un subconjunto de los MSD para los cuales sea posible encontrar un ciclo o camino de longitud máxima. Además, en este mismo contexto, se concluye que el cálculo de un ciclo de longitud $2 n-m$ contenido en un MSD es un problema NP-completo.

La construcción de un MSD mediante la descomposición en orejas nos ha permitido demostrar que si un MSD contiene más de un ciclo, entonces contiene al menos una cadena lineal. La última cadena lineal que se añade al MSD es una cadena lineal externa, que, al ser eliminada, produce otro MSD. Además, hemos demostrado que existe al menos una cadena lineal externa que no está contenida en el ciclo más largo del MSD. Este resultado podría ser de utilidad para investigar, en un futuro, si las cadenas lineales externas tienen una relación directa con los coeficientes del polinomio característico del MSD.

Finalmente, nos hemos acercado ligeramente a la demostración de la conjetura presentada en [7]. Probablemente, con la profundización del estudio en la líneas futuras, propuestas en los párrafos anteriores, sea posible encontrar una subclase de MSD para la que un conjunto de números reales cumplan con condiciones necesarias para ser coeficientes del polinomio característico correspondiente. 


\section{Bibliografía}

[1] Arcos-Argudo, M., García-López, J., Pozo-Coronado, L.M. Structure of cycles in Minimal Strong Digraphs, Discrete Applied Mathematics, 263 (2019), $35-41$.

[2] Arcos-Argudo, M., Lacalle, J., Pozo-Coronado, L.M. Structure of vertices and cycles in Minimal Strong Digraphs, (2019) - in review.

[3] Bang-Jensen, J., Gutin, G., Digraphs : theory, algorithms and applications, 2nd. ed., Springer, London, 2009.

[4] Berge, C. Graphes, North-Holland, Amsterdam, 1991.

[5] Chen, Z.B., Zhang, F.J., Bounds of the longest directed cycle length for minimal strong digraphs, Discrete Mathematics, 68 (1988), 9-13.

[6] García-López, J., Marijuán, C. Minimal strong digraphs, Discrete Math., 312 (2012), no. 4, 737-744.

[7] García-López, J., Marijuán, C., Pozo-Coronado, L.M. Structural properties of minimal strong digraphs versus trees, Linear Algebra and its Applications, 540 (2018), 203-220.

[8] R.P. Gupta, On basis digraphs, J. Combin. Theory 3 (1967) 16-24.

[9] R.A. Brualdi, H.J. Ryser, Combinatorial Matrix Theory, Cambridge University Press, New York, 1992.

[10] D.P. Geller, Minimally strong digraphs, Proc. Edinb. Math. Soc. 17 (2) (1970) 15-22.

[11] S. Hedetniemi, Characterizations and constructions of minimally 2-connected graphs and minimally strong digraphs, in: R.C. Mulin et al., (Eds.), Proc. 2nd 
Theory Louisiana Conference on Combinatorics, Graph Theory and Computing, Utilitas Mathematica, Winnipeg, 1971, pp. 257-282.

[12] G.A. Dirac, Minimally 2-connected graphs, J. Reine Angew. Math. 228 (1968) 204-216.

[13] M.D. Plummer, On minimal blocks, Trans. Amer. Math. Soc. 134 (1968) 85-94.

[14] M. Grötschel, On minimal strong blocks, J. Graph Theory 3 (1979) 213-219.

[15] K.K. Bhogadi, Decomposition and generation of minimal strongly connected digraphs, Master's Thesis, Univ. of Georgia, Athens, 1999.

[16] W.H. Cunningham, Decomposition of directed graphs, SIAM J. Algebr. Discrete Methods 3 (1982) 214-228.

[17] F. Zhang, X. Guo, Some properties of minimally strongly connected digraphs, J. Xinjiang Univ. Natur. Sci. 3 (1985) 1-6 (in Chinese).

[18] F. Harary, Graph Theory, Adisson-Wesley, 1969.

[19] R. Brualdi, M.B. Hedrick, A unified treatment of nearly reducible and nearly decomposable matrices, Linear Algebra Appl. 24 (1979) 51-73.

[20] Ausiello, Giorgio, Pierluigi Crescenzi, Giorgio Gambosi, Viggo Kann, Alberto Marchetti-Spaccamela, and Marco Protasi. Complexity and approximation: Combinatorial optimization problems and their approximability properties. Springer Science \& Business Media, 2012.

[21] Feder, Tomás, Rajeev Motwani, and Carlos Subi. Approximating the longest cycle problem in sparse graphs. SIAM Journal on Computing 31, no. 5 (2002): 1596-1607.

[22] Björklund, Andreas, Thore Husfeldt, and Sanjeev Khanna. Approximating longest directed paths and cycles. In International Colloquium on Automata, Languages, and Programming, pp. 222-233. Springer, Berlin, Heidelberg, 2004 .

[23] Gabow, Harold N., and Shuxin Nie. Finding a long directed cycle. ACM Transactions on Algorithms (TALG) 4, no. 1 (2008): 7. 
[24] Vishwanathan, Sundar. An approximation algorithm for finding a long path in Hamiltonian graphs. In Proceedings of the eleventh annual ACM-SIAM symposium on Discrete algorithms, pp. 680-685. Society for Industrial and Applied Mathematics, 2000.

[25] Monien, Burkhard. How to find long paths efficiently. In North-Holland Mathematics Studies, vol. 109, pp. 239-254. North-Holland, 1985.

[26] Fürer, Martin, and Balaji Raghavachari. Approximating the minimum degree spanning tree to within one from the optimal degree. In Proceedings of the third annual ACM-SIAM symposium on Discrete algorithms, pp. 317324. Society for Industrial and Applied Mathematics, 1992.

[27] Furer, Martin, and Balaji Raghavachari. Approximating the minimumdegree Steiner tree to within one of optimal. Journal of Algorithms 17, no. 3 (1994): 409-423.

[28] Karger, David, Rajeev Motwani, and Gurumurthy DS Ramkumar. On approximating the longest path in a graph. Algorithmica 18, no. 1 (1997): 82-98.

[29] Alon, Noga, Raphael Yuster, and Uri Zwick. Finding and counting given length cycles. Algorithmica 17, no. 3 (1997): 209-223.

[30] Rosen, K.H. and Krithivasan, K., 2012. Discrete mathematics and its applications: with combinatorics and graph theory. Tata McGraw-Hill Education.

[31] Minc, H., 1974. Irreducible matrices. Linear and Multilinear Algebra, 1(4), pp.337-342.

[32] London, D., 1999. Irreducible matrices with reducible principal submatrices. Linear algebra and its applications, 290(1-3), pp.257-266.

[33] Cvetkovic, D., Doob, M., Sachs, H, Spectra of graphs: theory and application, Academic Press, 1980.

[34] J.A. Bondy, U.S.R. Murty, Graph Theory, Springer, 2008.

[35] T. Gallai, Problem 15, in: M. Fiedler (Ed.), Theory of Graphs and Its Applications, Czech. Acad. Sci., 1964, p.161. 
[36] S. Bessy, S. Thomassé, Spanning a strong digraph with alpha cycles: a conjecture of Gallai, Combinatorica 27(6) (2007) 659-667.

[37] M. Las Vergnas, Sur les arborescences dans un graphe orienté, Discrete Math. 15 (1976) 27-39.

[38] S. Thomassé, Covering a strong digraph by $\alpha-1$ disjoint paths: a proof of Las Vergnas' conjecture, J. Combin. Theory Ser. B 83 (2001) 331-333.

[39] Torre-Mayo, J., Abril-Raymundo, M. R., Alarcia-Estévez, E., Marijuán, C. and Pisonero, M., The nonnegative inverse eigenvalue problem from the coefficients of the characteristic polynomial, EBL digraphs, Linear Algebra Appl. 426 (2007) 729-773.

[40] Page, L., Brin, S., Motwani, R. and Winograd, T. The PageRank citation ranking: Bringing order to the web. Stanford InfoLab. (1999).

[41] https://jglopez.etsisi.upm.es/MSC_Digraphs_Page/. 